\title{
Faithful representations with topographic maps
}

\author{
Marc M. Van Hulle \\ Laboratorium voor Neuro- en Psychofysiologie \\ K.U.Leuven \\ Campus Gasthuisberg \\ Herestraat \\ B-3000 Leuven, BELGIUM \\ Tel.: + 3216345961 \\ Fax: + 3216345993 \\ E-mail: marc@neuro.kuleuven.ac.be
}

\section{Request for reprints}

Marc M. VAN HULLE

\section{K.U.Leuven}

Laboratorium voor Neuro- en Psychofysiologie

Faculteit Geneeskunde

Campus Gasthuisberg

Herestraat

B-3000 Leuven

Belgium

Phone: + 3216345961

Fax: + 3216345993

E-mail: marc@neuro.kuleuven.ac.be

\section{Running title}

Faithful representations with topographic maps 


\title{
Faithful representations with topographic maps
}

\begin{abstract}
Topographic map algorithms that are aimed at building "faithful representations" also yield maps that transfer the maximum amount of information available about the distribution from which they receive input. The weight density (magnification factor) of these maps is proportional to the input density, or the neurons of these maps have an equal probability to be active (equiprobabilistic map). Since MSE minimization is not compatible with equiprobabilistic map formation in general, a number of heuristics have been devised in order to compensate for this discrepancy in competitive learning schemes, e.g. by adding a "conscience" to the neurons' firing behavior. However, rather than minimizing a modified MSE criterion, we introduce a new unsupervised competitive learning rule, called the kernelbased Maximum Entropy learning Rule (kMER), for topographic map formation, that optimizes an information-theoretic criterion directly. To each neuron a radially-symmetric kernel is associated, with a given center and radius, and the two are updated in such a way that the (unconditional) information-theoretic entropy of the neurons' outputs is maximized. We review a number of competitive learning rules for building equiprobabilistic maps. As benchmark tests for the faithfulness of the representations, we consider two types of distributions and compare the performances of these rules and kMER, for batch and incremental learning. As a first example application, we consider non-parametric density estimation where the maps are used for generating "pilot" estimates in kernel-based density estimation. The second application we envisage for kMER is "on-line" adaptive filtering of speech signals, using Gabor functions as wavelet filters. The topographic feature maps that are developed in this way differ in several respects from those obtained with Kohonen's Adaptive-Subspace SOM (ASSOM) algorithm.
\end{abstract}

Key words : topographic maps, equiprobabilistic maps, conscience learning, Gabor functions, wavelet filters, faithful representations, non-parametric density estimation 


\section{Introduction}

The Self-Organizing Map (SOM) algorithm (Kohonen, 1982,1984,1995) is a biologically-inspired unsupervised competitive learning scheme that, due to its inherent simplicity, has seen an impressive range of statistical applications. Its purpose is to develop a topology-preserving or neighborhood-preserving mapping from an input space $V \subseteq \Re^{d}$ onto a discrete lattice $A$, of $N$ formal neurons, with weights $\mathbf{w}_{1}, \ldots, \mathbf{w}_{N}, \mathbf{w}_{1}=\left[w_{11}, \ldots, w_{1 d}\right]$ and so on, and with a regular and fixed topology, in such a way that neighboring neurons code for neighboring positions in $V$-space (albeit that the inverse does not necessarily hold).

The SOM algorithm was originally conceived for non-parametric regression analysis (Ritter et al., 1992; Mulier and Cherkassky, 1995; Kohonen, 1995), but it has also been regarded as a discrete, non-parametric model of the input probability density insofar as it is not intended to model its fine-structure (Kohonen, 1995, p. 152). Contrary to what was originally assumed (Kohonen, 1984), the weight density at convergence, also termed the magnification factor in this context, is not a linear function of the (stationary) input density $p(\mathbf{v}), \mathbf{v}=\left[v_{1}, \ldots, v_{d}\right]$ : Ritter and Schulten (1986) have shown that, for a one-dimensional lattice (i.e. a chain), the weight density $p\left(\mathbf{w}_{i}\right)$ is proportional to $p(\mathbf{v})^{\frac{2}{3}}$, in the limit of an infinite density of neighbor neurons (continuum approximation, i.e. when the neighborhood range $\ll N$ but still $>1$ in practice). In the case of a discrete lattice, when the neighborhood range vanishes (zero-order topology), it is expected that, for large $N$, and for minimum mean squared error (MSE) quantization, the weight den-

sity will be proportional to $p^{\frac{1}{1+\frac{2}{d}}}(\mathbf{v})$ (Gersho, 1979; Zador, 1982; Kohonen, 1995). The connection between both limits was established by Ritter (1991), for a rectangular neighborhood function, and by Dersch and Tavan (1995) for arbitrary, monotonously decreasing neighborhood functions, both for the one-dimensional case.

\subsection{Equiprobabilistic maps}

Topographic maps have also been considered for applications where the weight density $p\left(\mathbf{w}_{i}\right)$ should in fact be proportional to the input density $p(\mathbf{v})$, or where the lattice neurons should have an equal probability to be active, i.e. an equiprob- 
abilistic map. From an information-theoretic point of view, such a map transfers the maximum amount of information available about the input distribution. Loosely speaking, this is what is usually meant by a "faithful representation" (Lin et al., 1997). Equiprobabilistic maps are desired in at least three applications: modeling sensory coding, non-parametric Blind Source Separation (BSS), and non-parametric density estimation e.g. for clustering or classification purposes. In section 2 we will review a number of learning rules for equiprobabilistic map formation.

When modeling sensory systems, it is often suggested that the goal of sensory coding is to produce a "sparse-distributed" code (for a review, see Field, 1994). In such a code, every neuron has the same probability of being active and that probability is low for a given neuron. Besides sensory coding, maps with linear magnification factors are also desired in order to be in line with the biological evidence (for a discussion, see Van Hulle, 1997c): a detailed analysis performed by Wässle and co-workers (Wässle et al., 1989) shows that there exists a close correspondence between the retinal ganglion cell density and the decrease in areal cortical magnification factor with eccentricity, so that it is not necessary to postulate an additional magnification in the lateral geniculate nucleus (LGN) and/or the visual cortex. In addition, the simple rule that the central representation follows the peripheral receptor or neural density may even be applicable not only to the visual system but to other sensory cortices as well (Wässle et al., 1989).

More recently, equiprobabilistic topographic maps were shown to be beneficial in non-parametric Blind Source Separation (BSS). When considering rectangular lattices, and linear- or mildly non-linear signal mixtures with sub-Gaussian (i.e. approximatively uniform) source densities, the output distribution of an equiprobabilistic map is factorizable along the lattice coordinates into statistically independent components (Kohonen et al., 1996; Pajunen et al., 1996; Moreau and Macchi, 1996). For each observed mixture $\mathbf{v}(t)$, the winning neuron is determined and its lattice coordinates are regarded as the quantized estimates of the source signals.

A more general application of equiprobabilistic maps is non-parametric density estimation: when the weight density is proportional to the input density, the converged map can be regarded as a non-parametric model of the input density 
and, in this way, it can be used for the BSS of source signals with super-Gaussian densities (Lin et al., 1997), and for density-based clustering or discriminant analysis (classification): equiprobabilistic maps allow for Bayesian borders to be found even when the classes have different probabilities of occurrence, contrary to the SOM algorithm where equal class occurrence is assumed (Kohonen, 1995, p. 111).

However, one could argue that for most density-based applications, it does not matter to have non-equiprobabilistic maps: one could always compute the winning frequencies of each neuron, probabilities $P_{i}$ in fact, and divide these by the volumes of the corresponding Voronoi regions, provided that they can be sufficiently accurately determined, to obtain an estimate of the input density, ${ }^{1}$ or one can use the $P_{i}$ 's as "pilot" probability estimates for a density estimation technique in which the neuron weights are supplemented with a fixed volume, fixed radius Gaussian kernel, scaled by $P_{i}$ (Parzen window technique, see Duda and Hart, 1973).

We will introduce a new unsupervised competitive learning rule for equiprobabilistic topographic map formation, called the kernel-based Maximum Entropy learning Rule (kMER), and assess its equiprobabilistic performance for batch and incremental ("on-line") learning. The first application we will explore is density estimation. Since kMER adapts not only the neuron weights but also the radii of the kernels centered at these weights, and since these radii are updated in such a way that they model the local input density at convergence, we can go beyond the Parzen window technique and use these radii directly, as they correspond to a pilot density estimate in fact, in variable kernel density estimation. We will conduct an empirical study in which the quality of the pilot density estimates obtained with kMER and the pilot probability estimates obtained with a number of unsupervised competitive learning rules, including the SOM algorithm, are evaluated from the perspective of kernel-based density estimation. The second application we will

\footnotetext{
${ }^{1}$ This should not be confounded with probability distribution estimation (i.e. cumulative distribution function or repartition function). The latter has been explored by Pagès (1993) in the context of smooth function integration. He showed that a lattice trained with the SOM algorithm, without the neighborhood function, can approximate the probability distribution of an input density $p(\mathbf{v})$ with infinite precision, when the number of Voronoi regions goes to infinity and provided that one keeps track of the winning frequencies during learning.
} 
consider is "on-line" adaptive filtering of speech signals by means of topographic feature maps, using Gabor functions as wavelet filters. Since we use the same speech example in fact, our approach can be clearly distinguished from Kohonen's Adaptive-Subspace SOM (ASSOM) algorithm (Kohonen, 1995; Kohonen et al., 1997). The mathematical details of kMER will be deferred to a follow-up article.

\section{Equiprobabilistic map formation}

There have been several attempts to build equiprobabilistic topographic maps with regular and fixed topologies and in this section, we will review a number of them. The most adopted strategy is to modify, with a heuristic, the standard unsupervised competitive learning (standard UCL) rule:

$$
\Delta \mathbf{w}_{i^{*}}=\eta\left(\mathbf{v}-\mathbf{w}_{i^{*}}\right)
$$

with $i^{*}$ the label of the neuron that wins the competition ("winner-takes-all", WTA $), i^{*}=\arg \min _{i \in A}\left\|\mathbf{w}_{i}-\mathbf{v}\right\|$ (nearest-neighbor rule), or the SOM algorithm:

$$
\Delta \mathbf{w}_{i}=\eta \Lambda\left(i, i^{*}, t\right)\left(\mathbf{v}-\mathbf{w}_{i}\right), \quad \forall i \in A,
$$

with $\Lambda$ the neighborhood function - a decreasing function of the distance between the lattice-space coordinates of $i^{*}$ and $i$ - and $\eta$ the learning rate. The RF's of the neurons are still defined by a (modified) nearest-neighbor rule, and thus are still non-overlapping and uniform Voronoi regions, and the learning rule yields a map which minimizes a (modified, or MSE-like) distortion metric. We call this the distortion-based approach. A different strategy is to maximize the informationtheoretic entropy of the map's outputs: when achieved, all neurons will have an equal probability to be active. We call this the information-based approach.

\section{$2.1 \quad$ Distortion-based}

Motivated originally by the concern that rarely used quantization regions do not sufficiently contribute to the minimization of the quantizer's overall distortion (due to quantization), several researchers were inspired by Grossberg's idea (1976) of adding a "conscience" to frequently winning neurons to feel "guilty" and to reduce their winning rate. It in effect implies that one is striving towards an equiprobable 
quantizer, namely, one in which every unit is used equally frequently. The same heuristic has also been adopted in combination with topographic map formation, and has also been extended towards equidistortion quantization. The competitive learning strategies that have been explored in conjunction with conscience learning belong to three broad categories: 1) the activation probabilities of the neurons are monitored and used for adjusting the distance metric in the nearest-neighbor rule, 2) the local distortion is monitored and used for adjusting either the learning rate in the weight update rule, or the distance metric in the nearest-neighbor rule, or 3) the activation probabilities are monitored together with the local distortions and the two are combined for adjusting the neurons' learning rates.

One of the learning rules that belong to the first category is Conscience Learning (DeSieno, 1988; Conscience 1): for each neuron $i$, the number of times it has won the competition $c_{i}$ is recorded, and a scaled version of this quantity, a bias in fact, is added to the distance metric used in the (modified) nearest-neighbor rule (following the format of Van den Bout and Miller, 1989):

$$
\left\|\mathbf{w}_{i^{*}}-\mathbf{v}\right\|+C c_{i^{*}} \leq\left\|\mathbf{w}_{i}-\mathbf{v}\right\|+C c_{i}, \quad \forall i \in A
$$

with $C$ the scaling factor (the "conscience factor"), and after which the "conscience" of the winning neuron $i^{*}$ is incremented: $c_{i^{*}} \leftarrow c_{i^{*}}+1$. The weight of the winning neuron is updated as in eq. (1). Hence, "conscience" is achieved by relating the definition of the winning neuron to its probability of being the winner (i.e. the activation probability). In its slightly modified version Conscience 2 (called TInMANN - The Integer Markovian Artificial Neural Network -, see Van den Bout and Miller, 1989), DeSieno's conscience mechanism is combined with Kohonen's SOM algorithm in order to produce topology-preserving maps. In yet another rule, called Frequency-Sensitive Competitive Learning (FSCL; Ahalt et al., 1990), the distance metric in the nearest-neighbor rule is scaled by the number of times neuron $i^{*}$ has been winning the competition:

$$
\left\|\mathbf{w}_{i^{*}}-\mathbf{v}\right\| \times c_{i^{*}} \leq\left\|\mathbf{w}_{i}-\mathbf{v}\right\| \times c_{i}, \quad \forall i \in A,
$$

and after which the conscience of the winning neuron is also incremented, and its weight vector updated using eq. (1). However, contrary to what was originally assumed (Ahalt et al., 1990, p. 283), FSCL does not achieve an equiprobable quantization: recently, Ahalt and a co-worker have shown that FSCL yields 
$p\left(\mathbf{w}_{i}\right) \propto p(\mathbf{v})^{\frac{2}{3}}$, in the one-dimensional case, and in the limit of an infinite number of neurons (Galanopoulos and Ahalt, 1996), a result that they considered to be favorable since it is closer to equiprobabilism than what is achieved by standard UCL (ibid. p. 754). A drawback of these conscience-based learning rules is that they all rely on some form of memory to keep track of the cumulative activity of the individual neurons, and this feature compromises their application in incremental learning.

Competitive learning schemes that belong to the second category exploit measures based on the local distortion error to equilibrate the neurons" "conscience". This idea has been pursued, in a heuristic manner, by adapting the neurons' learning rates during topographic map formation (Kim and Ra, 1995) and, in a more formal manner, by using a weighted distance metric in the nearest-neighbor rule, weighted by the (local) distortion error of each neuron, for building equidistortion quantizers (Chinrungrueng and Séquin, 1995; Ueda and Nakano, 1993). However, since equidistortion quantization is pursued, we will not further consider such learning rules.

An example of the third category is the learning scheme introduced Bauer, Der and Herrmann (1996) (BDH algorithm) for topographic formation under the continuum limit. It starts from the observation that the input density $p(\mathbf{v}) \propto p\left(\mathbf{w}_{i}\right) \times P_{i}$, with $P_{i}$ the probability that neuron $i$ is the winner given $p(\mathbf{v})$. The weight density $p\left(\mathbf{w}_{i}\right)$ is inversely proportional to the volume of the respective Voronoi region. Both $P_{i}$ and $p\left(\mathbf{w}_{i}\right)$ are approximated, in a heuristic way, by assuming that $P_{i}$ is related to the time interval between successive instants that neuron $i$ has been the winner, $\Delta_{i}$, and that Voronoi regions can be approximated by spheres with (averaged) radius $\left\|\mathbf{w}_{i}-\mathbf{v}\right\|^{d}$. The $\mathrm{BDH}$ algorithm then becomes, for the equiprobabilistic case:

$$
\Delta \mathbf{w}_{i}=\eta_{i^{*}} \Lambda\left(i, i^{*}, t\right)\left(\mathbf{v}-\mathbf{w}_{i}\right), \quad \forall i \in A,
$$

with

$$
\eta_{i^{*}}=\eta\left[\frac{1}{\Delta_{i^{*}}}\left(\frac{1}{\left\|\mathbf{w}_{i}-\mathbf{v}\right\|^{d}}\right)\right]^{\frac{1}{2}},
$$

and $d$ the effective dimensionality spanned by the data submanifold in the input 
space. ${ }^{2}$ Hence, "conscience" is achieved here by relating the weight update strength of the winner to its probability of being the winner.

A different strategy is to apply a competitive learning rule that minimizes the mean absolute error (MAE) between the input samples $\mathbf{v}$ and the $N$ weight vectors (also called the Minkowski metric of power one). The (almost) optimal learning rule related to this metric reads as follows (Kohonen, 1995, p. 121):

$$
\Delta \mathbf{w}_{i}=\eta \Lambda\left(i, i^{*}, t\right) \operatorname{sgn}\left(\mathbf{v}-\mathbf{w}_{i}\right), \quad \forall i \in A
$$

with $\operatorname{sgn}($.$) the sign function: \operatorname{sgn}(z)=1$ for $z>0,-1$ for $z<0$ and 0 for $z=0$. Based on the continuum limit analysis of Ritter and Schulten (1986), it can be shown that this rule yields $p\left(\mathbf{w}_{i}\right) \propto p(\mathbf{v})$ in the one-dimensional case. This property has been recently explored by Lin and co-workers (1997) but it did not always yield satisfactory equiprobable quantizations in practice. They attributed this shortcoming to the absence of a counting aspect in the learning rule, related to the activation probabilities. However, we feel that there is a more profound problem with eq. (7) in the discrete case, namely when $N$ is not very large, which is the case in practice: it can be formally proven that, under this circumstance, eq. (7) does not yield an equiprobable quantization for general, continuous input distributions (see Appendix 1).

Finally, we should note the following about distortion-based learning schemes. First, we know that aiming for an equiprobable quantization by adding a "conscience" can only be a heuristic since distortion minimization is mathematically speaking not equivalent to information-theoretic entropy maximization in the general case (Van Hulle and Martinez, 1993; Ueda and Nakano, 1993). Second, again owing to the presence of a "conscience", analytic criteria of what is being implemented are often lost, especially if one goes beyond the one-dimensional case: even though some improvement in achieving equiprobabilism can be observed, often no mathematical reason can be given for it (Bauer et al., 1996). Evidently, for some cases, this problem can be overcome when the input distribution is separable into one-dimensional distributions (Ritter and Schulten, 1989). Third, by the mere fact that a Voronoi tessellation is assumed, again as one goes beyond the onedimensional case, the quantization regions become convex polytopes, the volumes

\footnotetext{
${ }^{2}$ For a way to compute the effective dimensionality see e.g. Rojas, 1996, p. 405.
} 
of which are not easily determined. For this reason, activation probabilities have to be determined, but this in turn becomes increasingly difficult as the dimensionality of the input space increases ( $c f$. the curse of dimensionality). This could be kept under control if the effective dimensionality of the subspace spanned by the input samples is small, a strategy which is employed in the BDH algorithm. Fourth, distortion minimization becomes increasingly similar to information-theoretic entropy maximization when the dimensionality increases: in the high dimensionality limit, a minimum distortion quantizer becomes an equiprobable quantizer (Shannon, 1960).

\subsection{Information-based}

Instead of minimizing a (modified) distortion criterion, perhaps a more intuitive approach is to optimize an information-theoretic criterion directly. Ralph Linsker devised a global learning rule for topographic map formation in a probabilistic WTA network by maximizing the average mutual information (Shannon information rate) between the output and the signal part of the input, which was corrupted by noise (Linsker, 1989). For a given limit case, depending on the choice of the system parameters, the weight density becomes proportional to the input density. However, Linsker's rule also relies on some form of memory, which he called "historical information" - "conscience"-information in fact - to keep track of the cumulative activity of the individual neurons. Furthermore, the rule is relatively complex and has a limited capacity to unfold maps (ibid. p. 405).

Recently, we introduced a more direct way to equiprobabilistic topographic map formation, by maximizing the information-theoretic entropy of the map's outputs, using an "on-line" learning rule called the (lattice-based) Maximum Entropy learning Rule (MER) (Van Hulle, 1995,1997a). The lattice topology was rectangular and its dimensionality the same as that of the input space in which the lattice was developed. The quantization regions corresponded to the lattice quadrilaterals directly and hence, no Voronoi tessellation was assumed. We formally proved that in the one-dimensional case, MER yields an equiprobable quantization for any $N$ (Van Hulle, 1995), and that in the multi-dimensional case, it yields a quantization which will approximate an equiprobable one for large $N$ (Van Hulle, 1997a,b). A drawback of the equidimensionality assumption is that we cannot use MER 
e.g. for non-parametric regression- or dimensionality reduction purposes. Furthermore, the definition of quantization region is too complicated to be practical.

In the next section, we will relax the equidimensionality requirement and introduce a new, computationally-efficient unsupervised competitive learning rule for equiprobabilistic topographic map formation. This new learning rule is called kernel-based MER (kMER), since it shares the same spirit with MER - achieving equiprobabilistic neurons -, albeit that it is quite different in nature.

\section{Kernel-based maximum entropy learning}

Consider a lattice $A$ with a regular and fixed topology, and with arbitrary dimensionality $d_{A}$. To each neuron $i$ corresponds a radially-symmetric kernel $K\left(\mathbf{v}-\mathbf{w}_{i}, \sigma_{i}\right)$, with center $\mathbf{w}_{i}$ and radius $\sigma_{i}, e . g$. a Gaussian. Since the RF kernel is radially-symmetric, the cross-section with the neuron's activation threshold $\tau_{i}$ defines a circular- (or hyperspherical-, in general) $\mathrm{RF}$ region $S_{i}$, with radius $\sigma_{i}$, in $V$-space (Fig. 1A). If the input $\mathbf{v}$ falls in $S_{i}$, then we have a supra-threshold activation, and the threshold will be elevated, else we have a sub-threshold activation, and the threshold will be lowered. In order to formalize these events, we associate with each $S_{i}$ a code membership function:

$$
\mathbb{1}_{i}(\mathbf{v})= \begin{cases}1 & \text { if } \mathbf{v} \in S_{i} \\ 0 & \text { if } \mathbf{v} \notin S_{i}\end{cases}
$$

Since the RF regions can overlap (Fig. 1B), and since their radii are not defined by the other neuron weights, as in the Moody and Darken approach (Moody and Darken, 1988), we cannot simply rely on a "winner-takes-all" scheme, as in the original SOM algorithm, or on common vertices in the lattice quadrilaterals, as in MER, to pave the input space even when the lattice is in a topology-preserving state. In order to solve this problem, we define a fuzzy code membership function $\Xi_{i}($ Zadeh, 1965):

$$
\Xi_{i}(\mathbf{v})=\frac{\mathbb{1}_{i}(\mathbf{v})}{\sum_{k \in A} \mathbb{1}_{k}(\mathbf{v})}, \quad \forall i \in A,
$$

so that $0 \leq \Xi_{i}(\mathbf{v}) \leq 1$ and $\sum_{i} \Xi_{i}(\mathbf{v})=1$. Depending on the neural activation state, the weights $\mathbf{w}_{i}$ and radii $\sigma_{i}$ are adapted, in an iterative manner, by using two learning rules which together form the kernel-based Maximum Entropy 
learning Rule (kMER). The purpose of kMER is to generate, at convergence, a topographic map for which the supra-threshold activations of its neurons maximize the (unconditional) information-theoretic entropy (whence the rule's name).

Insert Fig. 1 about here.

Consider the update of the RF centers $\mathbf{w}_{i}$. Assume first that there is no neighborhood function (zero-order topology). We update $\mathbf{w}_{i}$ proportional to $\Xi_{i}$ and in the general direction of $\mathbf{v}($ Fig. 1B):

$$
\Delta \mathbf{w}_{i}=\eta \Xi_{i}(\mathbf{v}) \operatorname{Sgn}\left(\mathbf{v}-\mathbf{w}_{i}\right), \quad \forall i \in A,
$$

with $\operatorname{Sgn}($.$) the sign function taken componentwise. Second, as in the SOM al-$ gorithm, the critical factor in generating topology-preserving mappings is the use of a neighborhood function $\Lambda$ for updating the RF centers $\mathbf{w}_{i}$, i.e. a decreasing function of the distance, in lattice-space coordinates, between the supra-threshold activated neuron $i$ and its neighboring neurons. As a result, neighboring neurons cooperate and specialize for similar input signals and the lattice organizes into an orderly, topology-preserving state. With the neighborhood function present, eq. (10) becomes:

$$
\Delta \mathbf{w}_{i}=\eta \sum_{j \in A} \Lambda\left(i, j, \sigma_{\Lambda}\right) \Xi_{j}(\mathbf{v}) \operatorname{Sgn}\left(\mathbf{v}-\mathbf{w}_{i}\right), \quad \forall i \in A,
$$

with $\sigma_{\Lambda}$ the neighborhood range (in lattice space coordinates) of the neighborhood function $\Lambda($.$) , which is normalized to unity, \sum_{j \in A} \Lambda\left(i, j, \sigma_{\Lambda}\right)=1$. Apart from a cooperative element, there is also a competitive element in the learning process of the $\mathbf{w}_{i}$ 's: inputs that are shared by different neurons (shaded region in Fig. 1B) will lead to smaller weight updates. As a result, there will be a competitive element in the learning process since the RF centers will tend to be "pulled" apart by the unbalanced weight update strengths. Note that, in the absence of a neighborhood function, or when it has vanished, each RF center $\mathbf{w}_{i}$ is updated in such a way that its average corresponds to the (weighted by $\Xi_{j}$ ) median of the input samples that activate neuron $i$ : the sum of the weight updates $\Delta w_{i j}$ over the $j$ th input dimension will average zero when equal numbers of input samples produce positive and negative updates. (Note that there exists no unique definition of median in the higher-than-one-dimensional case.) 
Besides the centers $\mathbf{w}_{i}$ of the $\mathrm{RF}$ regions, we also update their radii $\sigma_{i}$. The idea is to adjust them in such a way that, at convergence, the probability for neuron $i$ to be active $P\left(\mathbb{1}_{i}(\mathbf{v})=1\right)=\frac{\rho}{N}, \forall i$, with $\rho$ a scale factor:

$$
\Delta \sigma_{i}=\eta\left(\frac{\rho_{r}}{N}\left(1-\mathbb{1}_{i}(\mathbf{v})\right)-\mathbb{1}_{i}(\mathbf{v})\right), \quad \forall i \in A,
$$

with $\rho_{r} \doteq \frac{\rho N}{N-\rho}$ (Fig. 1B). Since $P\left(\mathbb{1}_{i}(\mathbf{v})=1\right)=\frac{\rho}{N}$ at convergence, we have that:

$$
\tilde{p}\left(\mathbf{w}_{i}\right)=\frac{\rho}{N \operatorname{Vol}\left(\sigma_{i}\right)}
$$

with $\tilde{p}($.$) a density estimate, which can be located at the RF center, and Vol(.) the$ volume of the hypersphere, with radius $\sigma_{i}$, of neuron $i$ 's RF region. This capacity of the $\sigma_{i}$ to generate an initial ("pilot") estimate of the probability density $p(\mathbf{v})$ will be explored in the application section 6 in connection to variable kernel density estimation. Note that eq. (13) is reminiscent of the $k$ th-nearest-neighbor method (Silverman, 1992), with $k$ the number of samples, out of a fixed set of $M$ in total, that activate each one of $M$ regions used for obtaining the density estimate.

Finally, for $p(\mathbf{v})$ a stationary probability density, it can be proven that kMER converges, since a Liapunov function exists on which gradient descent is performed (Appendix 2), and that at convergence, the radius of each $\mathrm{RF}$ region will be such that $P\left(\mathbb{1}_{i}(\mathbf{v})=1\right)=\frac{p}{N}, \forall i \in A$ (Appendix 3 ). The results of a time-complexity analysis of kMER, for the case of batch learning, is given in Appendix 4.

\section{Distinction to Conscience Learning}

In Conscience 1 learning, as well as its variants Conscience 2 learning and FSCL, one keeps track of the number of times each neuron has won the competition, and in the $\mathrm{BDH}$ algorithm the last time each neuron has won the competition, and uses this information to update the neuron weights $\mathbf{w}_{i}$, and thus also the RF regions, since a Voronoi tessellation is assumed. The update is done in such a manner that every neuron will be active with (roughly) equal probability at convergence.

In kMER, the RF radii $\sigma_{i}$ keep track of another kind of information: they are estimates of equiprobabilistic portions of the input density. These estimates then influence the updates of the RF centers $\mathbf{w}_{i}$ by virtue of the overlap between the RF regions ( $c f$. fuzzy code membership function $\Xi_{i}$ - note that we do not assume a Voronoi tessellation). 


\section{Lattice Untangling Dynamics}

In order to show the learning dynamics of $\mathrm{kMER}$, we consider the standard case of mapping a two-dimensional input space $V$ onto a $24 \times 24$ planar lattice $A$. The inputs $\mathbf{v}=\left(v_{1}, v_{2}\right)$ are randomly and independently drawn from the two-dimensional uniform density $p(\mathbf{v})$ in the unit square $\left\{0 \leq v_{1}<1,0 \leq v_{2}<1\right\}$; the weights at time $t=0$ are chosen randomly in the same unit square. In particular, we re-consider the same random weight initialization and set of input samples previously used for the SOM algorithm and MER (Van Hulle, 1997b). In addition, we initialize the radii randomly by sampling homogeneously and independently the interval $(0,0.1]$. We use a small, fixed learning rate $\eta=0.001$ and perform "on-line" learning. ${ }^{3}$ We use the following Gaussian neighborhood function:

$$
\Lambda\left(i, i^{*}, t\right)=\exp \left(-\frac{\left(r_{i}-r_{i^{*}}\right)^{2}}{2 \sigma_{\Lambda}(t)^{2}}\right),
$$

with $r_{i}$ and $r_{i^{*}}$ the lattice coordinates of $i$ and $i^{*}$, and decrease its range $\sigma_{\Lambda}(t)$ in the following way:

$$
\sigma_{\Lambda}(t)=\sigma_{\Lambda 0} \exp \left(-2 \sigma_{\Lambda 0} \frac{t}{t_{\max }}\right)
$$

with $t$ the present time step, $t_{\max }$ the maximum number of time steps, and $\sigma_{\Lambda 0}$ the range spanned by the neighborhood function at $t=0$; we take $t_{\max }=2,000,000$ and $\sigma_{\Lambda 0}=12$.

The evolutions of the neuron weights and the corresponding RF regions are shown in Fig. 2 for $\rho_{r}=1$ in eq. (12) (left and right columns, respectively). Compared to MER and the SOM algorithm (Van Hulle, 1997b), the weight dynamics is quite different. The neuron weights at convergence are evenly distributed, as desired, but more importantly, the radii are also well adapted to achieve an equiprobable quantization since the standard deviation is $2.49 \times 10^{-4}$, with respect to $\frac{1}{N}$, when we normalize the probabilities so that their sum equals unity. The standard deviations for MER and the SOM algorithm were for this case $3.18 \times 10^{-4}$ and $6.08 \times 10^{-4}$,

\footnotetext{
${ }^{3}$ Note that, theoretically, we should decrease $\eta$ to zero at a suitable rate, e.g. $\eta=\frac{1}{t}$ (Ljung, 1977). However, since we use a small but fixed $\eta$, for reasons of comparison with our earlier work, we will verify convergence empirically. In that case, $\eta$ should at least be an order of magnitude smaller than the smallest radius of the RF regions (see Appendix 5).
} 
respectively. What is particularly interesting is the re-arrangement of the circles after 100,000 iterations: the overlap between the circles decreases and hence, they become more closely packed. This is a consequence of the competitive element in the learning process, which is aimed at reducing the overlap, but it seems only to be a weak force in the topographic map formation process since it is only effective when the neighborhood range has practically vanished. The effect of $\rho_{r}$ on the overlap is demonstrated in Fig. 3: for $\rho_{r}=0.5$, the RF regions seem to be scattered, whereas for $\rho_{r}=2$, they seem to be arranged in a more regular fashion.

Insert Figs. 2 and 3 about here.

\section{Equiprobabilistic map performance}

Since kMER is aimed at building equiprobabilistic maps, we will consider two types of input densities as benchmark tests for comparing kMER's performance with that of a number of other algorithms of which some directly perform, or can be made suitable for topographic map formation: the SOM algorithm, the (lattice-based) Maximum Entropy learning Rule (MER) (Van Hulle, 1997a), the BDH algorithm (Bauer et al., 1996), the Conscience 1 rule (DeSieno, 1988) and its modified version the Conscience 2 rule (TInMANN; Van den Bout and Miller, 1989), and FSCL (Ahalt et al., 1990). We consider two cases: incremental and batch learning. For a time-complexity analysis of these learning schemes, for the case of batch learning, we refer to Appendix 4.

\subsection{Incremental learning}

Consider as a first test-case the quadrimodal "product" distribution shown in (Fig. 4A), i.e. an unbounded density function with bounded support in the unit square $[-1,1)^{2}$, which is difficult to quantize since it consists of four heavily skewed but disjunct modes separated by sharp transitions (discontinuities). The analytic equation of this density function is, in the first quadrant, $\left(-\log v_{1}\right)\left(-\log v_{2}\right)$, with $\left(v_{1}, v_{2}\right) \in[0,1)^{2}$, i.e. the two-dimensional "product" distribution, and so on. For all learning algorithms, we consider the planar lattice of the previous simulation, with 
the same number of time steps $t_{\max }$, neighborhood cooling scheme, and weightand radius initialization procedures. The details of the simulations are given in Appendix 6.

After the learning process has terminated, we quantify the equiprobabilistic performance of each learning algorithm. We proceed in two ways. First, we consider the root mean squared error (RMSE) between the desired activation probability of each neuron $i, \frac{1}{N}$ (we take $\rho=1$ in kMER), and the actually obtained one, $P_{i}=\frac{1}{M} \sum_{\mu=1}^{M} \mathbb{1}_{i}\left(\mathbf{v}^{\mu}\right)$, for a given test set of $M=100 k$ novel samples:

$$
R M S E=\sqrt{\frac{\sum_{i=1}^{N}\left(\frac{1}{N}-P_{i}\right)^{2}}{N}} .
$$

Second, we consider the information-theoretic entropy $I$ :

$$
I=-\sum_{i=1}^{N} P_{i} \log _{2} P_{i}, \quad \text { (bits) }
$$

whereby the maximum attainable performance equals $I_{\max }=\log _{2} N=9.17$ bits since $N=576$ in our simulations. The results are summarized in Table 1.

\subsection{Batch learning}

The next test-case is taken from (Herbin et al., 1996): three equally-probable Gaussians with standard deviation 0.2 and centered at $(-0.4,-0.3),(0.4,-0.3)$, and $(0 ., 0.3)$. The underlying density function is now bounded and has an unbounded support. Rather than incremental learning, as done in the previous example, we now consider batch learning on a fixed set of $M=900$ samples drawn from the three Gaussians (Fig. 6). The lattice dimensions, neighborhood function and initializations are similar ( $t_{\max }=10,000$ epochs $)$.

We again determine the RMSE and $I$ but now for a test set of $M=9000$ samples. The results are summarized in Table 2. For kMER we will quantify the effect of the dimensionality $d$ in section 6.2 .2 .

Insert Tables 1 and 2 about here. 


\section{Non-parametric density estimation}

Once the learning process has terminated, we can use the converged lattice for building a non-parametric model of the input density $p(\mathbf{v})$. This is our first application. Basically two approaches can be adopted in practice. The first is to determine the winning frequencies of all neurons and assume fixed, equally sized volumes for the corresponding regions. This will lead to the Parzen window technique and the allocation of fixed-radii kernels to obtain the density estimate $\tilde{p}$, i.e. fixed kernel density estimation. A second approach is to do the inverse and determine the region volumes in such a way that they yield equal winning frequencies (i.e. equiprobabilism). This leads to variable kernel density estimation and this case applies in particular to kMER. We start with a brief discussion of the two approaches and later in this section, we will consider techniques for determining the "optimal" degree of smoothness of the resulting density estimates.

\section{Fixed kernel estimate}

We first compute the winning frequencies $P_{i}$ of each neuron and then allocate kernels with equal (i.e. fixed) radii at the neuron weights. The fixed kernel density estimate is then directly obtained using the classical Parzen window technique:

$$
\hat{p}(\mathbf{v})=\sum_{i=1}^{N} P_{i} K\left(\mathbf{v}-\mathbf{w}_{i}\right)=\sum_{i=1}^{N} P_{i} \frac{\exp \left(-\frac{\left\|\mathbf{v}-\mathbf{w}_{i}\right\|^{2}}{2\left(\rho_{s}\right)^{2}}\right)}{Z},
$$

with $K$ a fixed volume, fixed radius kernel (here, a Gaussian) with center $\mathbf{w}_{i}$ and $Z$ a normalizing factor. The parameter $\rho_{s}$ controls the degree of smoothness of the resulting density estimate. Several techniques are available with which $\rho_{s}$ can be determined in an "optimal" manner. We will consider one such technique in section 6.1. Finally, it is important to note that the fixed-kernel technique, together with an "optimal" smoothness technique, can be applied to any weight distribution, be it equiprobabilistic or not, provided that one determines the winning frequencies.

\section{Variable kernel estimate}

Since the lattice generated by kMER not only consists of the neuron weights but also the radii of the neurons' $\mathrm{RF}$ regions, with the radii adapted to the local input density, we can go beyond the Parzen window technique (for an explanation, see 
further). We can cast density estimation into a format which is similar to the one used for variable kernel density estimation (VK) (Silverman, 1992):

$$
\widehat{p_{\rho s}}(\mathbf{v})=\sum_{i=1}^{N} \frac{K\left(\mathbf{v}-\mathbf{w}_{i}, \rho_{s} \sigma_{i}\right)}{Z_{i}}=\sum_{i=1}^{N} \frac{\exp \left(-\frac{\left\|\mathbf{v}-\mathbf{w}_{i}\right\|^{2}}{2\left(\rho_{s} \sigma_{i}\right)^{2}}\right)}{Z_{i}}
$$

when Gaussian kernels are used, with $\rho_{s}$ a factor with which the radii $\sigma_{i}$ can be

scaled, and $Z_{i}$ a proper normalizing factor so that $\frac{\int_{V} K(., .) d \mathbf{v}}{Z_{i}}=\frac{1}{N}$. It can be easily verified that both $\rho_{s}$ and $\rho_{r}$ control the degree of smoothness of the resulting non-parametric density estimate, and we will deal with their choice in a moment.

\section{Going beyond Parzen window technique}

The learning schemes of section 5 can only provide a "pilot" estimate in terms of probabilities $P_{i}$, and not in terms of densities. The classic VK method relies on a pilot density estimate $\tilde{p}$ in order to determine the $\sigma_{i}$ 's in eq. (19), which in turn has to be provided by another density estimation technique, such as the ( $k$ th-)nearestneighbor method. With kMER, a pilot density estimate is obtained (eq. (13)) but, more importantly, it can be directly expressed in terms of the kernel radii used in the VK method: as a result of this, we can simply exchange the RF radii $\sigma_{i}$ in kMER for kernel radii in the VK method. By virtue of this intimate connection between the radius of a RF region and the kernel radius, VK density estimation becomes an obvious application of kMER.

\section{Distinction to classic VK approach}

In classic VK, a kernel is allocated at every input sample and the sum directly yields the final density estimate. Hence, if we have a batch of $M$ samples, then $M$ kernels will be used. With kMER, we can allocate only a restricted number of kernels, typically $N \ll M$, and the location and width of these kernels will be the subject of learning. In this way, the density estimate can be gradually build up, following the presentation of each input sample (on-line learning) and, thus, not only based on fixed sample sets which have to be available before density estimation can start. As more samples become available, the weights and radii determined with kMER will gradually converge and the density estimate eq. (19) will gradually reach its final state. (Note that there is also a computational- as well as a memory space advantage attached to it.) 


\subsection{Automatic choice of smoothing parameter}

\section{Fixed kernel estimate}

Silverman (1992) reviews a range of techniques to determine the "optimal" degree of smoothing, such as the subjective graph method, the least-squares crossvalidation method, the likelihood cross-validation method, and the test graph method, to name a few. The most widely-used technique, and which allows for a completely automatic procedure, is the least-squares cross-validation method. It can be applied to both fixed and variable kernel density estimation, at least in principle. The idea is to choose $\rho_{s}$ in such a way that the integrated squared error:

$$
\int_{V}(\hat{p}(\mathbf{v})-p(\mathbf{v}))^{2} d \mathbf{v}
$$

is minimized. This is equivalent to minimizing the score function:

$$
\mathcal{M}_{0}\left(\rho_{s}\right)=\int_{V} \hat{p}^{2}(\mathbf{v}) d \mathbf{v}-\frac{2}{N} \sum_{i} \hat{p}_{-i}\left(\mathbf{w}_{i}\right)
$$

with $\hat{p}_{-i}$ the density estimate constructed from all data points except $\mathbf{w}_{i}$ (leave-oneout method). The integral can be approximated as: $\frac{1}{N^{2} h} \sum_{i, j} K^{(2)}\left(\mathbf{w}_{i}-\mathbf{w}_{j}\right)$, with $h$ the kernel bandwidth and $K^{(2)}$ the convolution of the kernel with itself. When the kernel is Gaussian, and when fixed kernel density estimation is performed, it can be shown that, asymptotically, when $N \rightarrow \infty$, the theoretically best choice of $\rho_{s}$ is obtained (Stone, 1984). Despite of its strong attractiveness, cross-validation may yield poor results. When a nearest-neighbor classification of the input samples is performed, the input samples become discretized and, even though the lattice may be fine-grained, it can be shown that, under mild conditions, cross-validation can choose the degenerate smoothness value $\rho_{s}=0$.

\section{Variable kernel estimate}

If we turn to variable kernel density estimation, as we are doing for kMER, the theoretical foundation for choosing $\rho_{s}$ becomes less strong and the score function eq. (20) cannot be simplified. In addition, the use of a Gaussian becomes rather inappropriate here because of the computationally expensive evaluation of the exponential in the Gaussian kernel definition. For these reasons, we derive a different strategy for choosing $\rho_{s}$ and $\rho_{r}$. 
Consider first the case where $\rho_{r}$ is fixed. Assume that the pilot estimate eq. (13) is quite accurate so that we can select a $\rho_{s}$-value that minimizes the MSE between, on one hand, the density estimate $\widehat{p_{\rho_{s}}}$, for a given $\rho_{s}$-value and located at the $\mathbf{w}_{i}$ 's, and on the other hand, the pilot estimate eq. (13) (i.e. without using kernels):

$$
\rho_{s}=\arg \min _{\rho_{s}} M S E\left(\widehat{p_{\rho_{s}}}, \tilde{p}\right) \doteq \arg \min _{\rho_{s}} \frac{\sum_{i=1}^{N}\left(\widehat{p_{\rho_{s}}}\left(\mathbf{w}_{i}\right)-\tilde{p}\left(\mathbf{w}_{i}\right)\right)^{2}}{N} .
$$

The underlying hypothesis is that:

$$
\min _{\rho_{s}} M S E\left(\widehat{p_{\rho_{s}}}, \tilde{p}\right) \approx \min _{\rho_{s}} M S E\left(\widehat{p_{\rho_{s}}}, p\right)
$$

at the observation sites $\mathbf{w}_{i}$. Unfortunately, this procedure generates too low $\rho_{s^{-}}$ values (usually $\ll 1$ ) to be reliable: the pilot estimate is not subject to the same sort of smoothing effects as the final estimate $\widehat{p_{\rho_{s}}}$. This suggests that we should consider a fixed kernel estimate $\widehat{p^{*}}$ and use it for obtaining an "optimal" $\rho_{s}$ value. We start by allocating fixed kernels at the $\mathbf{w}_{i}$ 's, with their radii equal to the average kernel radius obtained with kMER:

$$
\widehat{p^{*}}(\mathbf{v})=\sum_{i=1}^{N} \frac{K\left(\mathbf{v}-\mathbf{w}_{i}, \sigma^{*}\right)}{Z}
$$

with $\sigma^{*} \doteq\left\langle\sigma_{i}\right\rangle_{A}$ and $Z$ the usual normalizing factor. We then define the following MSE metric:

$$
\operatorname{MSE}\left(\widehat{p_{\rho_{s}}}, \widehat{p^{*}}\right)
$$

so that the "optimal" $\rho_{s}$-value (with respect to the fixed kernel case) then becomes:

$$
\rho_{s o p t}=\arg \min _{\rho_{s}} M S E\left(\widehat{p_{\rho_{s}}}, \widehat{p^{*}}\right) \doteq \arg \min _{\rho_{s}} \frac{\sum_{i=1}^{N}\left(\widehat{p_{\rho_{s}}}\left(\mathbf{w}_{i}\right)-\widehat{p^{*}}\left(\mathbf{w}_{i}\right)\right)^{2}}{N} .
$$

The whole procedure is derived from the following heuristic. Since for the MSE between the true density $p$ and the estimates obtained with the variable- and fixed kernel techniques holds that: $\sum_{i}\left(\widehat{p_{\rho_{s}}}\left(\mathbf{w}_{i}\right)-p\left(\mathbf{w}_{i}\right)\right)^{2} \leq \sum_{i}\left(\widehat{p^{*}}\left(\mathbf{w}_{i}\right)-p\left(\mathbf{w}_{i}\right)\right)^{2}$, in the vicinity of the optimal degree of smoothing of the variable kernel estimate, the MSE performance of the fixed kernel estimate can be considered as an upper bound for the variable kernel performance since in the former case, we assume that all radii are equal and, thus, that the distribution is locally uniform. Hence, by minimizing the MSE for the fixed kernel estimate, the maximal MSE error made for the variable kernel estimate will be lower. In order to optimize $\rho_{s}$, estimates for the 
$p\left(\mathbf{w}_{i}\right)$ are needed: these are provided by the variable kernel estimate and they are assumed to be better than those provided by the fixed kernel estimate: $\sum_{i}\left(\widehat{p^{*}}\left(\mathbf{w}_{i}\right)-\right.$ $\left.p\left(\mathbf{w}_{i}\right)\right)^{2} \approx \sum_{i}\left(\widehat{p^{*}}\left(\mathbf{w}_{i}\right)-\widehat{p_{\rho s}}\left(\mathbf{w}_{i}\right)\right)^{2}$. In other words, we perform a least-squares crossvalidation by constructing fixed- and variable kernel density estimates, and by minimizing the discrepancy between the two, i.e. $\min M S E\left(\widehat{p^{*}}, \widehat{p_{\rho_{s}}}\right)$, over $\rho_{s}$, for obtaining the optimal value, $\rho_{\text {s opt }}$. We then repeat the whole procedure along the $\rho_{r}$-axis to obtain the optimal mix $\left(\rho_{r o p t}, \rho_{s o p t}\right)$.

\subsubsection{Examples}

Consider again the two examples of section 5. We start with the quadrimodal product distribution (Fig. 4A) and incremental learning. The overall degree of smoothing $\left(\rho_{\text {ropt }}, \rho_{\text {sopt }}\right)$ is determined both by minimizing $\mathcal{M}_{0}$ and by using our technique, for the sake of comparison. Consider first the results of optimizing for $\rho_{s}$, given $\rho_{r}=1$ (Fig. $5 \mathrm{~A}$ ). The theoretically-optimal value is located at 3.5 (minimum of thick continuous line), i.e. with reference to the analytic equation of the original distribution. This optimum is rather closely approximated by the two cross-validation techniques, since they both yield $\rho_{\text {sopt }}=3.1$ (all when optimizing in steps of $\Delta \rho_{s}=0.1$ ). What is also interesting is that the minimum is quite deep in both cases, and this in turn facilitates the location of the "optimal" $\rho_{\text {sopt }}$ with a minimum number of evaluations. The next step is to repeat this optimization procedure for different $\rho_{r}$ values (Fig. 5B). The theoreticallyoptimal mix $\left(\rho_{\text {ropt }}, \rho_{\text {sopt }}\right)=(2 ; 2.1)$, when optimizing in steps of $\Delta \rho_{r}=1$, and the corresponding MSE equals $5.75 \times 10^{-2}$. Our cross-validation technique yields $\left(\rho_{\text {ropt }}, \rho_{\text {sopt }}\right)=(2 ; 2.9)$ (minimum of thin continuous line $)$, also with $\Delta \rho_{r}=1$, and a MSE of $5.76 \times 10^{-2}$, also with reference to the analytic equation; the resulting density estimate is shown in Fig. 4B. The usual cross-validation technique, $\min \left(\mathcal{M}_{0}\right)$, fails to yield an acceptable mix (dashed line): when looking for a minimum in $\mathcal{M}_{0}$, either the degenerate solution $\rho_{r} \rightarrow 0$ is obtained, or one which smoothens the four modes of the original distribution into a single mode, $\rho_{r} \gg$.

We now continue with the three equally-probable Gaussians (Fig. 6) and batch learning. Consider first the results of optimizing $\rho_{s}$, given $\rho_{r}=17.5$ (Fig. 5C). The theoretically-optimal value is located at 1.3 (minimum of thick continuous line), and this is again quite close to the value obtained with our technique, $\rho_{\text {sopt }}=1.5$ 
(minimum of thin continuous line) (both when optimizing in steps of $\Delta \rho_{s}=0.1$ ). The usual cross-validation technique, $\min \left(\mathcal{M}_{0}\right)$, yields the degenerate solution $\rho_{s} \rightarrow 0$ (dashed line). When also optimizing for $\rho_{r}$ (Fig. 5D), with $\Delta \rho_{r}=2.5$, the theoretically-optimal mix is found to be $(15 ; 1.4)$ (minimum of thick continuous line) and the "optimal" mix obtained with our technique is $(17.5 ; 1.5)$ (minimum of thin continuous line). Finally, there is a serious problem with the usual crossvalidation technique: even when we restrict the range over which an optimum is to be sought, a strategy which is suggested when $\mathcal{M}_{0}$ is expected to yield a degenerate solution (Silverman, 1992), or when keeping $\rho_{s}$ constant, e.g. equal to 1 , and optimizing for $\rho_{r}$, the usual cross-validation technique yields no acceptable minimum (dashed line).

Insert Figs. 4,5 and 6 about here.

\subsection{Density estimation performance}

\subsubsection{Incremental learning}

We continue with the quadrimodal product distribution (Fig. 4A) and use it as a benchmark for comparing the density estimation performance of kMER and the other learning algorithms that we have considered in section 5 .

In order to obtain density estimates for the maps generated with MER, FSCL, the two Conscience rules, and the SOM and BDH algorithms, we apply the Parzen window technique eq. (18) and determine the degree of smoothing by minimizing $\mathcal{M}_{0}$ (since the kernel radii are all equal), except for the $\mathrm{BDH}$ algorithm: since it yields the degenerate solution $\rho_{s} \rightarrow 0$, we take $\rho_{s}=0.075$, namely a value in the range of that of the other learning rules just mentioned. For the sake of comparison, we also consider the classic VK method (for implementation details, see again Appendix 6).

The results are summarized in Table 3 and listed in terms of the MSE as well as the Kullback-Leibler distance (cross-entropy), both with reference to the analytic equation of the original distribution. We conclude that, of all learning rules listed, kMER is the best rule, and that its performance is close to that of the classic VK 
method. It thus yields the most "faithful representation". The next best rule is MER, followed by the Conscience 2 rule.

Insert Table 3 about here.

\subsubsection{Batch learning}

If we turn again to the three equally-probable Gaussians example, and use batch learning, instead of incremental learning, then we obtain the results listed in Table 4. For VK, we use all 900 samples available in the training set. Furthermore, since we are now considering a fixed data set, we can also apply the Parzen window technique eq. (18) with the $P_{i}$ 's taken from a histogram obtained by uniformly partitioning the unit square $[-1,1]^{2}$ into $N=25 \times 25$ equally-sized bins; $P_{i}$ is then the proportion of the $M=900$ samples falling in each bin. The width of the Gaussian kernels is selected by minimizing $\mathcal{M}_{0}$ (results labeled "Parzen/histogram"). We conclude from Table 4 that: 1) kMER is again the best learning rule, and it closely matches VK's performance, and 2) that the performances of the other rules are relatively similar (all with reference to the MSE).

Insert Table 4 about here.

Finally, we wish to quantify the effect of the dimensionality $d$ of the input space on the equiprobabilistic performance of kMER. To this end, we use the same set of 900 samples but add a number of additional dimensions to them: these are obtained by sampling homogeneously and independently a zero-mean Gaussian with standard deviation 0.2. By consequence, when the dimensionality increases, the input space becomes gradually more sparsely covered by the 900 samples. We initialize randomly the neuron weights by sampling the uniform distribution $(-1,1]^{d}$. As a performance measure, we again use the RMSE eq. (16). The results are: $4.02 \times 10^{-5}$ for $d=2,3.81 \times 10^{-5}$ for $d=4,3.85 \times 10^{-5}$ for $d=10$, and $3.55 \times 10^{-5}$ for $d=20$. Note that the $d=2$ case corresponds to Table 4 . Hence, kMER's performance does not seem to degrade in the range of input space dimensions listed. 


\section{Topographic feature map formation: application to speech signals}

Up to now, we have only focussed on the occurrence of the input samples $\mathbf{v} \in V$, and we have built a faithful representation of the stationary probability density $p(\mathbf{v})$ from which they were drawn. However, since kMER adapts kernels, both in location and in range, we can supplement these kernels with a specific structure in $V$-space, so that they become filters or "feature detectors". We are then interested in the occurrence of certain features in these input samples: we wish to build a faithful representation for the distribution of these features by using a topographic feature map. Specifically, we will consider speech signals and adapt kMER for the purpose of obtaining a faithful representation of the energy spectrum of the speech source. The adaptation that we will introduce is two-fold: 1) the accommodation of graded neural activation levels instead of binary ones (cf. eq. (8)), and 2) the definition of each neuron as a linear subspace since we want these activation levels to be phase-invariant. The results described in this section are therefore only valid for the adapted version of kMER.

As an example, we use speech signals taken from the TIMIT database (1990), which are sampled at $f_{\text {samp }}=16 \mathrm{kHz}$. Each input vector $\mathbf{v}=\left[v_{1}, \ldots, v_{d}\right]$ now becomes a time sequence of $d$ samples or $T$ time units (in $s$ ) long. As a temporal filter, we use the (one-dimensional) Gabor function (Gabor, 1946): i.e. a sine wave, with a particular (radian) frequency and phase, modulated by a Gaussian kernel with a specific range, which in turn defines the filter's tuning width in the frequency domain. In kMER's format, the radian frequency of the $i$ th filter (in $\frac{\mathrm{rad}}{\mathrm{s}}$ ) corresponds to the kernel center $w_{i}$, and the filter's range in the temporal domain (in $s$ ) corresponds to the kernel radius $\sigma_{i}$. Since a Gabor function with an arbitrary phase can be expressed as a combination of a sine- and a cosine Gabor function, we let each neuron $i$ correspond to a linear subspace spanned by this quadrature pair:

$$
\begin{aligned}
& G_{s}^{i}\left(j, w_{i}, \sigma_{i}\right)=\sin \left(w_{i}\left(\frac{T j}{d}-\frac{T}{2}\right)\right) \exp \left(-\frac{\left(\frac{T j}{d}-\frac{T}{2}\right)^{2}}{2 \sigma_{i}^{2}}\right) \\
& G_{c}^{i}\left(j, w_{i}, \sigma_{i}\right)=\cos \left(w_{i}\left(\frac{T j}{d}-\frac{T}{2}\right)\right) \exp \left(-\frac{\left(\frac{T j}{d}-\frac{T}{2}\right)^{2}}{2 \sigma_{i}^{2}}\right), \quad j=1, \ldots, d .
\end{aligned}
$$


In the same manner as Adelson and Bergen (1985), we define the activation of neuron $i$ as a measure for the local energy:

$$
A c t_{i}=\left(\sum_{j=1}^{d} G_{s}\left(j, w_{i}, \sigma_{i}\right) v_{j}\right)^{2}+\left(\sum_{j=1}^{d} G_{c}\left(j, w_{i}, \sigma_{i}\right) v_{j}\right)^{2}, \quad \forall i \in A,
$$

which also corresponds to the squared length of the orthogonal projection of $\mathbf{v}$ onto the quadrature pair and, thus, a phase-invariant quantity. This implies that, instead of having sub- and supra-threshold activations, as in our original kMER equations, we now have graded responses. We will also adapt kMER in such a way that the energy spectrum can be regarded as the equivalent of the probability density function; the details of the adapted version of kMER are presented in appendix 7. Based on the neural activations $A c t_{i}$, and given the overlaps between the kernels, the $w_{i}$ 's and $\sigma_{i}$ 's are updated following the presentation of each time sequence $\mathbf{v}$. All this can be done by using information available in the time domain only. As a result, instead of each neuron being active with an equal probability $\frac{\rho}{N}$ at convergence, as before, the activations $A c t_{i}$ will now be, on average, equal to $\frac{\rho}{N}$ times the average energy present in the original time sequences. ${ }^{4}$

Before we train our lattice, we first select a wav file from the TIMIT database $(\mathrm{dr} 1 / \mathrm{fcjf0} / \mathrm{sa} 2)$. The speech signal is shown in Fig. $7 \mathrm{~A}$ : it is 34509 samples or $\approx$ $2.16 \mathrm{~s}$ long. The corresponding energy spectrum is displayed in Fig. 7B, normalized between 0 and 3 , for the sake of representation. The energy spectrum has a number of distinct peaks, which makes it difficult to represent with a restricted number of filters. From the wav file we randomly select 284 time sequences of length $d=480$ samples (i.e. $30 \mathrm{~ms}$ ). We consider $N=10$ neurons and randomly initialize their weights in the interval $\left(0, \frac{2 \pi f_{s a m p}}{4}\right]$ (i.e. half the range spanned by the radian frequency domain), and the radii in the interval $\left(0, \frac{2 \pi f_{\text {samp }}}{40}\right]$ (i.e. one-twentieth of that range). After the presentation of a time sequence, we calculate the neuron activations and update (all) the kernel centers and radii; we take $\eta=0.05$ and $\rho=2$, and no neighborhood function. We continue training until 500,000 iterations have elapsed.

\footnotetext{
${ }^{4}$ The latter is done in order to put our radii update scheme in a unified format: unlike probability densities, energy spectra do not necessarily have unit volumes.
} 
Insert Figs. 7 and 8 about here.

The evolution of the kernel centers $\mathbf{w}_{i}$ is shown in Fig. 7C. The (cosine) Gabor functions obtained at convergence are displayed in Fig. 8, in the time domain, and the corresponding kernels in Fig. 7D in the radian frequency domain. The sum of the kernels is shown in Fig. 7E (thick line), together with the original spectrum (thin line); we have normalized the sum in such a way that the two energy spectra have equal surface areas. We have also indicated the result for $\rho=8$ in Fig. 7F. Based on these results, we conclude that the kernel centers are grouped according to the location and the surface areas of the peaks in the energy spectrum. This is also the case for the kernel centers when $\rho=8$ (result not shown). Furthermore, $\rho$ again controls the degree of smoothing but now in terms of the energy spectrum estimate (Fig. 7E vs. F). Since for $\rho=2$, the kernels are relatively narrow in the energy spectrum, the kernels that belong to different peaks tend to have only limited overlaps compared to the $\rho=8$ case. As a result of this, the distribution of the average neural activations has a larger spread than in the $\rho=8$ case: the means of the average neural activations, with the average taken over the last 100 iterations, and the corresponding standard deviations are 1.82 and 0.66 amplitude $^{2} \mathrm{rad} / \mathrm{s}$ for $\rho=2$, and 4.96 and 0.25 for $\rho=8$. However, on the other hand, the $\rho=2$ case yields a better spectral estimate. When using our smoothing procedure, the "optimal" spectral estimate is obtained for $\rho=2$, which also corresponds to that of the theoretically-optimal case (both when optimizing $\rho$ in steps of 0.25 ). Finally, since the kernel centers are strongly grouped, with almost equal kernel widths, we can easily detect this grouping and decrease the redundancy of the representation by keeping one kernel for each group and by adjusting the height of that kernel according to the number of kernels it is supposed to replace. In this way, four quadrature Gabor pairs are retained with a comparable spectral estimation quality. 


\section{Discussion}

We have introduced a new unsupervised competitive learning rule, called the kernel-based Maximum Entropy learning Rule (kMER), for building faithful representations of input probability densities by using equiprobabilistic topographic maps with fixed and regular topologies. Our approach differs from that of the original SOM algorithm (Kohonen, 1982,1984,1995) by the criterion optimized (distortion minimization vs. entropy maximization) and by the type of Receptive Fields (RF's) used (non-overlapping, Voronoi-based RF's vs. overlapping, kernelbased RF's). In a similar way, it also differs from the learning rules that combine distortion minimization with a "conscience" to adjust the neurons' winning rates. We have reviewed a number of conscience-type of learning rules and assessed their abilities to generate equiprobabilistic maps.

Since the RF's overlap in kMER, in order to pave the input space with our kernels, we had to come up with a new definition of competitive learning. We have defined a fuzzy code membership function $\Xi_{i}$ that is a measure of the probability that neuron $i$ belongs to the subset of the activated neurons, rather than just the probability that neuron $i$ is active; the update of the kernel center of neuron $i$ is then proportional to $\Xi_{i}$. The definition of our fuzzy code membership function is quite different from the one used in the fuzzy-clustering literature (see Rose et al., 1990), where each datum is associated in probability with each cluster: when no assumptions are made about the data distribution, then the "fuzzy membership in clusters" appears in the form of the softmax function, i.e. a normalized exponential, normalized with respect to all clusters. This type of membership function has also been recently used by Graepel et al. (1997), when deriving their Soft Topographic Vector Quantizer (STVQ).

Besides the kernel centers, we also adjust the kernel radii with kMER. The learning rules that are commonly used in combination with kernels, usually in the form of Radial Basis Functions (RBF's), assume that the kernel radii are chosen in an ad hoc fashion (Moody and Darken, 1988; Fritzke, 1994). However, in kMER, the radii are simply considered as additional weight components (thresholds in fact) and they are individually-adapted to the local sample density, so as to yield an equiprobabilistic topographic map at convergence. When kMER would be used 
for regression purposes, the connection with RBF networks or more generally, with HyberBF networks, using limited numbers of kernels (Poggio and Girosi, 1990), becomes clear. The individually-adapted kernels then perform local smoothing of the interpolation function, depending on the local sample density, instead of depending on the overall (MSE) regression performance (Poggio and Girosi, 1990) or the likelihood of the input samples (Xu and Jordan, 1993; Nowlan, 1990; for a recent discussion, see Bishop, 1995). This application will be explored in the future. The real-world application that we have explored in this article, and which takes advantage of the fact that kMER adapts kernels, is the formation of topographic feature maps for speech signals. To this end, we have extended our rule to the case where one is interested, not only in the occurrence of input events, but also in the presence of certain features in them. We have shown how kMER develops a faithful representation for the distribution of these features by using Gabor functions as RF kernels.

Due to this extension of kMER, the connection with the Adaptive-Subspace SOM (ASSOM) algorithm, for the formation of Gabor-like wavelet filters (Kohonen, 1995; Kohonen et al., 1997), comes to mind. Evidently, the two approaches have some similarities. They both yield topographic feature maps that represent statistical representations which refer to different temporal events generated by the signal source rather than to the global stationary statistical properties of that source, as is done in a Principal Component Analysis (PCA). Also, both learning algorithms rely on quadrature filter pairs in order to obtain phase-invariant representations. However, apart from these similarities, kMER is quite different in several respects. First, the adaptation of the weight vectors in the ASSOM algorithm is aimed at maximizing the length of the projection vector onto the linear subspace associated with each neuron; in the case of kMER, the weights are updated so that they become the weighted medians of the neurons' RF regions. Furthermore, unlike kMER, the weights spanning each subspace in the ASSOM algorithm need to be normalized after each update. Second, and more importantly, the ASSOM algorithm relies on the identification of a unique "representative winner" for each set of subsequent time sequences (called episode) in order for the neighborhood around that winning neuron to learn the general linear combination of particular adjacent time sequences and, in this way, to achieve (temporal) invariance. Hence, in 
the ASSOM algorithm, a whole sequence is "memorized" before a "representative winner" can be selected and the weights updated. In kMER, no episodes or representative winners are needed: instead, for each time sequence, all neuron weights (and radii) are updated (they all are "winners" so to speak). Third, the ASSOM algorithm is aimed at developing Gabor-like wavelet filters, whereas kMER uses Gabor functions directly and adjusts their parameters. Hence, the two approaches are fundamentally different. One could then argue that the ASSOM algorithm is in a better position to generate wavelet filters that are tuned to the statistical properties of the signal source. In fact, this argument touches upon the pros and cons of parametric vs. non-parametric modeling approaches. We have shown that $\mathrm{kMER}$ is able to reconstruct the energy spectrum of the signal source since the activation of each neuron represents a measure for the local signal energy in the neuron's frequency band; in the case of the ASSOM algorithm, it seems that the neuron activations attempt to code for the amplitude spectrum, which is less "peaked" than the energy spectrum, but in any case, no spectral reconstruction results have been shown in the ASSOM literature. More importantly, irrespective of the type of spectrum that is approximated, or the optimization criterion that is adopted, the ASSOM algorithm is clearly intended to develop Gabor-like wavelets, and several precautions were taken in order to achieve that goal. Since the ASSOM algorithm can not adapt the parameters of the Gabor function directly, it faces a much higher-dimensional parameter space than kMER and hence, a much more complex implementation. Unlike kMER, the input sequences are high-pass filtered, in order to enhance their high-frequency content, and normalized before they are applied to the ASSOM. In addition, in order to favor the development of localized (wavelet) filters, the pre-processed sequences are windowed with a Gaussian but, more importantly, the width of this Gaussian is under external control. Furthermore, as noted by Kohonen, in order to obtain a good filter distribution, a dissipation term has to be added to the weights (p. 1330 Kohonen et al., 1997), and the different learning parameters have to be controlled rather closely during the learning phase (see Fig. 5.15 in Kohonen, 1995; or Fig. 4 in Kohonen et al., $1997)$.

Finally, there is the role played by our scale factor $\rho$ since it distinguishes, in a fundamental way, our approach from that of e.g. Bauer and co-workers (1996): 
their scale factor controls the weight density of the map (i.e. the magnification factor) and, by virtue of the Voronoi tessellation that is assumed, also the activation probability distribution of the neurons. In Linsker's approach, the ratio between lateral inhibition and forward activation can be modulated: when it is large, the weight density becomes proportional to the input density, and thus corresponds to an equiprobabilistic map, or when it is small, the weight density becomes a power law of the input density, and the map is no longer equiprobabilistic. In our case, the choice of $\rho$ does not alter the activation probability distribution, since the neurons of the map always have the same activation probabilities, but rather it controls the degree of smoothing in the topographic map or, more precisely, the degree of overlap between the RF regions. The major motivation for choosing $\rho>1$ is that, in data bound applications, there are simply not enough training data available to account for detailed density models $\left(c f . \rho_{r}\right.$ in our batch learning example vs. our incremental learning example). The obvious alternative is to apply regularization or smoothing by increasing the degree of overlap between the RF regions in a manner similar to (variable) kernel smoothing (Silverman, 1992). In the opposite case, when $\rho<1$, we will obtain a more sparse but, in essence, a (low-)equiprobabilistic representation of the input space - a property which is desired in "sparse-distributed" sensory coding schemes (for a review, see Field, 1994). Olshausen and Field (1996) further explored this idea and introduced a non-parametric strategy in order to develop Gabor-like sparse-distributed representations, by maximizing the sparseness of these representations directly. However, they had to assume sparse-distributed, sampled Gabor functions as training inputs or whitened/lowpass filtered images of natural scenes, which in fact consist of sparse-distributed spectra. Although we are able to exchange our kernels with Gabor functions, it still remains an open question if we are able to build sparse-distributed representations with kMER. 


\section{Acknowledgments}

The author wishes to thank Helge Ritter, Technische Fakultät, Bielefeld University, Germany, for sharing his insights on the magnification factor of topographic maps, Gilles Pagès, Université de Paris 6, France, for pointing out the connection between probability distribution estimation, smooth function integration and the SOM algorithm, Michael Herrmann, Max-Planck-Institut für Strömungsforschung, Göttingen, Germany, for helpful discussions on the BDH algorithm, and Temujin Gautama, Laboratorium voor Neuro- en Psychofysiologie, K.U.Leuven, Belgium, for his assistance in developing the speech application. M.M.V.H. is a research associate of the Fund for Scientific Research - Flanders (Belgium) and is supported by research grants received from the Fund for Scientific Research (G.0185.96), the National Lottery (Belgium) (9.0185.96), and the Flemish Regional Ministry of Education (Belgium) (GOA 95/99-06). 


\section{Appendix 1: MAE minimization vs. equiprobable}

\section{quantization}

Consider a regular $N$-point scalar quantizer which maps a scalar-valued input signal $v \in V$ onto one of $N$ quantization levels (weights), $w_{1}, w_{2}, \ldots, w_{N}$, which in turn are trained using eq. (7) so as to minimize the MAE due to quantization. We assume that the input probability density $p(v)$ is continuous and stationary, and that the weights are ordered, in increasing order, at convergence (i.e. an ordered, one-dimensional chain). Furthermore, we assume that the neighborhood function is an even (symmetric) function with range $\ll N$. Since this learning rule assumes a nearest-neighbor classification, for assigning inputs to neuron weights, the quantization intervals are delimited by boundary points which in turn are defined as the midpoints between two successive weights: $\theta_{i}=\frac{w_{i}+w_{i+1}}{2}, i=1, \ldots, N-1$.

Proposition 1: An equiprobable quantization of the real line cannot be a stable configuration of the ensemble averaged learning rule eq. (7) for general, continuous input distributions $p(v)$.

Proof:

Assume the inverse and claim that eq. (7) yields an equiprobable quantization for a finite number of quantization levels $N$ : the probability that a quantization interval is active, $P(i) \doteq \int_{\theta_{i-1}}^{\theta_{i}} p(v) d v=\frac{1}{N}, i=2, \ldots, N-1$, and $P(1) \doteq \int_{-\infty}^{\theta_{1}} p(v) d v=\frac{1}{N}$, $P(N) \doteq \int_{\theta_{N-1}}^{\infty} p(v) d v=\frac{1}{N}$. Let's further concentrate on weight $w_{i}$, without loss of generality. Putting the ensemble average of eq. (7) equal to zero we have that, at convergence:

$$
\begin{aligned}
\int_{\theta_{i-1}}^{\theta_{i}} \operatorname{sgn}\left(v-w_{i}\right) p(v) d v & +\int_{-\infty}^{\theta_{i-1}} \Lambda\left(i, i^{*}\right) \operatorname{sgn}\left(v-w_{i}\right) p(v) d v \\
& +\int_{\theta_{i}}^{\infty} \Lambda\left(i, i^{*}\right) \operatorname{sgn}\left(v-w_{i}\right) p(v) d v=0
\end{aligned}
$$

where the neighborhood function $\Lambda$ remains present (since the continuum limit is approximated). This can be rewritten as:

$$
-\int_{\theta_{i-1}}^{w_{i}} p(v) d v-\int_{-\infty}^{\theta_{i-1}} \Lambda\left(i, i^{*}\right) p(v) d v+\int_{w_{i}}^{\theta_{i}} p(v) d v+\int_{\theta_{i}}^{\infty} \Lambda\left(i, i^{*}\right) p(v) d v=0,
$$

since we assume that the weights are ordered. This can be further rewritten as:

$$
-\sum_{i^{*}<i} \Lambda\left(i, i^{*}\right) \frac{1}{N}+\sum_{i^{*}>i} \Lambda\left(i, i^{*}\right) \frac{1}{N}-\int_{\theta_{i-1}}^{w_{i}} p(v) d v+\int_{w_{i}}^{\theta_{i}} p(v) d v=0 .
$$


Now since $\Lambda$ is assumed to be an even function, with range $\ll N$, we have that, except for the weights in the vicinity of the lattice border, the first two terms in the previous equation are identical, hence:

$$
\int_{\theta_{i-1}}^{w_{i}} p(v) d v=\int_{w_{i}}^{\theta_{i}} p(v) d v
$$

or, in other words, $w_{i}$ is the median of its quantization interval: $\int_{\theta_{i-1}}^{w_{i}} p(v) d v=$ $\int_{w_{i}}^{\theta_{i}} p(v) d v=\frac{1}{2 N}$. As a result, the real line is partitioned into $2 N$ equiprobable and disjunct quantization intervals defined between two successive boundary/weight and weight/boundary pairs. Now since the boundary points are the midpoints of two successive weights, the two successive intervals they delimit are of equal length. Hence, when scanning the real line, we encounter successive pairs of equal length intervals that are in addition equally probable. Clearly, this cannot be a stable configuration for an arbitrary input distribution and, thus, the initial claim is false. QED.

In the limit of high-resolution quantization, i.e. when the number of quantization intervals goes to infinity, the interval lengths become so small that we can ignore the distinction between midpoint and centroid (median), and the fact that we have successive pairs of equal-length intervals. Under this condition only, eq. (7) yields an equiprobable quantization.

\section{Appendix 2: Convergence of kMER}

We give a sketch of the proof that kMER eqs. $(11,12)$ converges; the full proof will be detailed elsewhere since it is quite tedious. We first need a number of definitions.

Since the code membership function $\mathbb{1}_{i}(\mathbf{v})$ is not differentiable at its border, we replace it by the following "smooth" function:

$$
\mathcal{S}_{i}\left(\mathbf{v}, \mathbf{w}_{i}, \sigma_{i}\right) \doteq \frac{1}{1+\exp \left(-\beta \frac{\sigma_{i}^{2}-\left\|\mathbf{v}-\mathbf{w}_{i}\right\|^{2}}{2}\right)}, \quad \forall i \in A,
$$

i.e. the usual sigmoid function, and $\beta$ the gain. The arguments of this function are chosen in such a way that it approaches $\mathbb{1}_{i}(\mathbf{v})$ when $\beta \rightarrow \infty$. The fuzzy code membership function $\Xi_{i}$ eq. (9) is re-defined as:

$$
\Xi_{i}^{\beta}(\mathbf{v})=\frac{\mathcal{S}_{i}(\mathbf{v})}{\sum_{k \in A} \mathcal{S}_{k}(\mathbf{v})}, \quad \forall i \in A,
$$


and the following "local error" is ascribed to each neuron $i$ :

$$
e_{i}^{\beta}=e_{w i}^{\beta}+e_{\sigma i}^{\beta}, \quad \forall i \in A,
$$

with

$$
\begin{aligned}
e_{w i}^{\beta} & =\sum_{j=1}^{N} \Lambda\left(i, j, \sigma_{\Lambda}\right) \Xi_{j}^{\beta}(\mathbf{v})\left|\mathbf{v}-\mathbf{w}_{i}\right|, \\
e_{\sigma i}^{\beta} & =\int_{0}^{\sigma_{i}}-\frac{\rho_{r}}{N}+\left(1+\frac{\rho_{r}}{N}\right) \mathcal{S}_{i}\left(\mathbf{v}, \mathbf{w}_{i}, \sigma_{i}^{*}\right) d \sigma_{i}^{*} .
\end{aligned}
$$

The averaged learning rules then become, after some algebraic manipulations:

$$
\begin{aligned}
& <\Delta \mathbf{w}_{i}>_{V}=-\eta \lim _{\beta \rightarrow \infty}<\frac{\partial e_{w i}^{\beta}}{\partial \mathbf{w}_{i}}>_{V}=\eta<\sum_{j=1}^{N} \Lambda\left(i, j, \sigma_{\Lambda}\right) \Xi_{j}(\mathbf{v}) \operatorname{Sgn}\left(\mathbf{v}-\mathbf{w}_{i}\right)>_{V}, \\
& <\Delta \sigma_{i}>_{V}=-\eta \lim _{\beta \rightarrow \infty}<\frac{\partial e_{\sigma i}^{\beta}}{\partial \sigma_{i}}>_{V}=\eta<\frac{\rho_{r}}{N}-\left(1+\frac{\rho_{r}}{N}\right) \mathbb{1}_{i}(\mathbf{v})>_{V}, \quad \forall i \in A .(38)
\end{aligned}
$$

In order to show that a Liapunov function exists, on which gradient descent is performed, for small enough $\eta$, it can be shown that the derivatives of both average learning rules form a symmetric matrix:

$$
\mathbf{H}=\left[\begin{array}{ll}
\mathbf{H}^{1} & \mathbf{H}^{2} \\
\mathbf{H}^{3} & \mathbf{H}^{4}
\end{array}\right]
$$

with $\mathbf{H}^{1}=\left[H_{j i}^{1} \doteq \frac{\partial \mathbf{F}_{i}}{\partial \mathbf{w}_{j}}\right], \mathbf{H}^{2}=\left[H_{j i}^{2} \doteq \frac{\partial \mathbf{G}_{i}}{\partial \mathbf{w}_{j}}\right], \mathbf{H}^{3}=\left[H_{j i}^{3} \doteq \frac{\partial \mathbf{F}_{i}}{\partial \sigma_{j}}\right]$, and $\mathbf{H}^{4}=$ $\left[H_{j i}^{4} \doteq \frac{\partial \mathbf{G}_{i}}{\partial \sigma_{j}}\right]$, with $\mathbf{F}_{i} \doteq<\sum_{j=1}^{N} \Lambda\left(i, j, \sigma_{\Lambda}\right) \Xi_{j}(\mathbf{v}) \operatorname{Sgn}\left(\mathbf{v}-\mathbf{w}_{i}\right)>_{V}$ and $\mathbf{G}_{i} \doteq$ $<\frac{\rho_{r}}{N}-\left(1+\frac{\rho_{r}}{N}\right) \mathbb{1}_{i}(\mathbf{v})>_{V}$. Since the global matrix $\mathbf{H}$ eq. (39) is symmetric - it is a Hessian - a Liapunov function exists on which the average learning rules perform gradient descent.

\section{Appendix 3: Equiprobable quantization with kMER}

Proposition 2: Given a statistically-stationary input probability density $p(\mathbf{v})$, the kernel radii developed with averaged kMER are guaranteed to define neurons with an equal probability to be active at convergence.

Proof: We have the following equilibrium point:

$$
\begin{aligned}
<\Delta \sigma_{i}>_{V}=0 & =<\left(\frac{\rho_{r}}{N}\left(1-1_{i}(\mathbf{v})\right)-1_{i}(\mathbf{v})\right)>_{V} \\
& =\frac{\rho_{r}}{N}\left(1-P_{i}\right)-P_{i}=\frac{\rho_{r}}{N}-\left(1+\frac{\rho_{r}}{N}\right) P_{i}, \quad \forall i
\end{aligned}
$$


with $P_{i}$ the probability that neuron $i$ is active, given $p(\mathbf{v})$, and from which follows that:

$$
P_{i}=\frac{\rho_{r}}{N+\rho_{r}}=\frac{\rho}{N}, \quad \forall i
$$

since $\rho_{r} \doteq \frac{\rho N}{N-\rho}$. Now since $\frac{\rho}{N}$ is a constant, independent of the lattice index $i$, and since $P_{i}$ is a monotonically increasing function of the kernel radius of neuron $i$, eq. (42) is the sole solution resulting from $\left\langle\Delta \sigma_{i}\right\rangle_{V}=0$, Furthermore, again since $P_{i}$ is a monotonically increasing function of $\sigma_{i}$, the solution is a stable one: for $P_{i}=\frac{\rho_{r}}{N+\rho_{r}}+\epsilon$, with $\epsilon$ a disturbance from the equilibrium condition $\left\langle\Delta \sigma_{i}\right\rangle_{V}=0$, we have that $\left\langle\Delta \sigma_{i}>_{V}=-\left(1+\frac{\rho_{r}}{N}\right) \epsilon<0\right.$ for $\epsilon<0$. Hence, we are guaranteed to obtain a set of equiprobable neurons at convergence. QED.

\section{Appendix 4: Time-complexity analysis}

In order to compare the computational cost of the different learning algorithms we use, we perform a time-complexity analysis for batch learning on $M$ samples. We differentiate between the number of assignments $(=)$, additions/subtractions $(+/-)$ and multiplications/divisions $(* /:)$ required by the algorithms and retain the highest-order terms. The multiplications/divisions are responsible for the largest part of the computational cost. The results are summarized in Table 5 and were obtained as follows.

Each learning scheme has a neighborhood function $\Lambda$ except for the Conscience 2 rule where two random numbers are generated in order to implement the effect of a neighborhood function (Van den Bout and Miller, 1989). For the learning schemes which possess a genuine neighborhood function, in order to avoid calculating this function in the innermost loop ( $N M d$-nested loop), namely where the weight updates $\Delta w_{i j}$ are accumulated, we calculate the contribution of the neighborhood function before this loop is executed. All random numbers needed in the learning schemes were generated with the RAN1 routine (Press et al., 1990) but the computational cost does not count in the final results since it is not used in the innermost loops. For kMER and the Conscience 2 rule, we consider the sign operation to be equivalent to one multiplication/division. Since the fuzzy code membership function in kMER does not depend on the dimension $d$, it can 
be kept outside the innermost loop as well where it is multiplied with the neighborhood function. We did not include the results for MER since we could only provide a rough indication of its complexity: $\mathcal{O}\left(d !^{d}\right)$. This is due to the fact that the code membership function of each lattice quadrilateral results in calculating the volumes of $d !+2 d !=3 d ! d$-dimensional tetrahedra (Fig. A1B in Van Hulle, 1997b). In summary, all learning schemes have a time-complexity which is on the order of $\mathcal{O}(N M d)$, except for MER.

Insert Table 5 about here.

\section{Appendix 5: Choice of learning rate}

The learning rate $\eta$ should be small enough for the average RF centers at convergence to represent the (weighted) medians of the samples that activate the respective neurons, and for the average radii to activate these neurons in an equiprobabilistic manner (section 3). However, since the RF regions are changing - both their centers and radii are updated - the set of samples that can activate a given neuron $i$ at a given time $t\left(i . e .\left\{\mathbf{v} \mid \mathbf{v} \in S_{i}(t)\right\}\right)$ might change over time. Hence, the median of the set of all samples that can activate neuron $i$ in this way, e.g. over a certain time span, might not correspond to the average RF center. (This reasoning also holds for the radius.) Hence, the learning rate should be small enough for the average discrepancy between the set median and the average RF center to be smaller than the magnitude of the fluctuations around this average discrepancy. We expect this to happen when $\eta$ is at least an order of magnitude smaller than the smallest radius of all RF regions: in this way, the set of all samples that activate a given neuron will largely correspond to the set of samples which originate from the "average" RF region, and the average RF center will be in agreement with the median of the sample set. Note that the average discrepancy and magnitude of the fluctuations (standard deviation) can be verified experimentally.

In order to obtain a lower bound estimate on the smallest radius one can expect at convergence, a reasonable number of samples should be drawn from the training set, say $M$, and for each sample the $k$-th smallest Euclidean distance between that 
sample and the other $(M-1)$ samples should be determined, with $\left\lfloor M \times \frac{\rho}{N}\right\rfloor \leq$ $k \leq\left\lfloor M \times \frac{\rho}{N}\right\rfloor+1$ and $\lfloor z\rfloor$ the integer part of $z$. This can be achieved with the Quicksort algorithm using about $(2+2 \ln 2) M \log _{2} k$ comparisons. The minimum Euclidean distance obtained in this way, for all samples, is then a lower bound on the smallest RF radius.

\section{Appendix 6: Implementation details}

The details of the incremental learning simulations are as follows. We use the same $N=24 \times 24$ planar lattice for each algorithm, and in addition, for the SOM algorithm and MER, we use the same Gaussian neighborhood function, cooling scheme and random weight initialization as in the kMER simulation; for the BDH algorithm we use $m=0.5$ (Bauer et al., 1996), to achieve equiprobabilism (the product distribution is separable), and a rectangular neighborhood function, $\Lambda\left(r_{i}, r_{i^{*}}\right)=1$, iff $\left\|r_{i}-r_{i^{*}}\right\| \leq \sigma_{\Lambda}$, else $\Lambda=0$, of which we keep the radius $\sigma_{\Lambda}=2$ fixed since we need to work under the continuum limit. We supplement FSCL (Ahalt et al., 1990) and the Conscience 1 rule (DeSieno, 1988) with a Gaussian neighborhood function in order to obtain topographic maps. Furthermore, we take the "conscience factor" in the Conscience 1 rule $C=1$ : higher values lead to increasingly unstable results, with the neuron weights all grouped around the distribution mean, certainly for the $C=10$ case, which was recommended by DeSieno. For the Conscience 2 rule (TInMANN; Van den Bout and Miller, 1989), we take $C=2$, as recommended by Van den Bout and Miller, and for their two other parameters we take $R_{1}=\operatorname{random}\left(d_{T}\right)$ and $R_{2}=\operatorname{random}\left(d_{T}\right)$, with random(.) the uniform distribution between 0 and $d_{T}$, and $d_{T}$ the "threshold distance"; we let $d_{T}$ evolve over time in exactly the same way as we are doing for $\sigma_{\Lambda}$. Finally, we take $\eta=0.015$ for the SOM algorithm, FSCL and the Conscience 1 rule, and $\eta=0.001$ for MER, the Conscience 2 rule and the $\mathrm{BDH}$ algorithm: in this way, the intensity with which the neuron weights vary at convergence is similar for all learning rules, including kMER.

For the variable kernel method (VK), we take the sensitivity parameter $\alpha=\frac{1}{2}$ (Breiman et al., 1977); the pilot estimate is determined by using the ( $k$ th-)nearestneighbor technique, with $k=\sqrt{M}$. The MSE, with reference to the analytic 
equation of the original distribution, decreases with increasing training set size $M$ but seems to level off around $M=10000$ : MSE $=5.92 \times 10^{-2}$ for $M=2000$, $5.57 \times 10^{-2}$ for $M=5000$, and $5.35 \times 10^{-2}$ for $M=10000$. Hence, we further concentrate on the $M=10000$ case.

\section{Appendix 7: kMER for topographic feature maps}

Since we now use kernels with a specific temporal structure - so that they become temporal filters in fact -, and since we are interested in representing the energy spectrum of the signal source with these filters, we need to adapt kMER so that adaptive filtering becomes possible at run-time. We proceed in the following way. Consider first the kernel weights $w_{i}$. Remember that the purpose of the $\Xi_{j} S g n$ term in eq. (11) is to let $w_{i}$ converge to the weighted median of the RF region $S_{i}$, weighted by the overlap between $S_{i}$ and the other $\mathrm{RF}$ regions $S_{j}$. We now split $S_{i}$ into two subranges: $S_{i}^{+}$, which corresponds to the range $\left[w_{i}+\sigma_{i}^{\omega}\right]$, and $S_{i}^{-}$which corresponds to $\left[w_{i}-\sigma_{i}^{\omega}\right]$. (Note that $\sigma_{i} \doteq \frac{1}{\sigma_{i}^{\omega}}, c f$. the time scaling property of the Fourier transform.) We then replace the $\Xi_{j} S g n$ term in eq. (11) by the difference of two terms: the integrals of the energy spectra in $S_{i}^{+}$and $S_{i}^{-}$, respectively. Evidently, we can proceed in the spectral domain and determine these integrals numerically. However, since we wish to use information available in the time domain only, we take for the integral in $S_{i}^{+}$a linear estimate, $I_{i}^{+}$, located at $w_{i}+\sigma_{i}^{\omega} / 2$, which is obtained by using the neurons' activations only:

$$
I_{i}^{+}=\sigma_{i}^{\omega}\left(A c t_{i}+\frac{A c t_{i+1} / 2-A c t_{i} / 2}{w_{i+1}-w_{i}} \frac{\sigma_{i}^{\omega}}{2}\right)
$$

and a similar equation for $I_{i}^{-}$. We also have to take into account the overlap between the RF regions: we determine the proportion of, e.g., $S_{i}^{+}$, for which an overlap exists with the RF range $S_{j}$ of neuron $j, \forall j \neq i$. Hence, the term $\Xi_{i}$ in eq. (9) again becomes a ratio, but now for $S_{i}^{+}$and $S_{i}^{-}$separately, of the interval length $\sigma_{i}^{\omega}$ divided by $\sigma_{i}^{\omega}$ and the sum over all $j \neq i$ of the proportion of $\sigma_{i}^{\omega}$ that neuron $i$ shares with neuron $j$. Let's call these ratios $f_{i}^{+}$and $f_{i}^{-}$, respectively. Hence, the $w_{i}$ 's are updated, in an incremental manner, as follows:

$$
\Delta w_{i}=\eta \sum_{j \in A} \Lambda\left(i, j, \sigma_{\Lambda}\right) \operatorname{sgn}\left(f_{i}^{+} I_{i}^{+}-f_{i}^{-} I_{i}^{-}\right), \quad \forall i \in A
$$


Second, similar to eq. (12), the radii are updated in such a way that, at convergence, the average activation of each neuron will be equal:

$$
\Delta \sigma_{i}^{\omega}=\eta \operatorname{sgn}\left(\frac{\rho E}{N}-A c t_{i}\right), \quad \forall i \in A,
$$

with $E$ the energy in the original waveform $\mathbf{v}$ ( $c f$. Parseval's theorem). 


\section{References}

Adelson, E.H., and Bergen, J.R. (1985). Spatiotemporal energy models for the perception of motion. J. Opt. Soc. Am. A, 2, 284-299.

Ahalt, S.C., Krishnamurthy, A.K., Chen, P., and Melton, D.E. (1990). Competitive learning algorithms for vector quantization. Neural Networks, 3, 277-290.

Bauer, H.-U., Der, R., Herrmann, M. (1996). Controlling the magnification factor of self-organizing feature maps. Neural Computation, 8, 757-771.

Bishop, C. (1995). Neural networks for pattern recognition. Oxford: Clarendon.

Breiman, L., Meisel, W., and Purcell, E. (1977). Variable kernel estimates of multivariate densities. Technometrics, 19, 135-144.

Chinrungrueng, C., and Séquin, C.H. (1995). Optimal adaptive $k$-means algorithm with dynamic adjustment of learning rate. IEEE Trans. Neural Networks, 6, $157-169$.

Dersch, D.R., and Tavan, P. (1995). Asymptotic level density in topological feature maps. IEEE TNN, 6, 230-236.

DeSieno, D. (1988). Adding a conscience to competitive learning. Proc. IEEE International Conference on Neural Networks, San Diego, Vol. I, pp. 117-124.

Duda, R.O., and Hart, P.E. (1973). Pattern classification and scene analysis. New York: Wiley.

Field, D.J (1994). What is the goal of sensory coding? Neural Computation, 6, 559-601.

Fritzke, B. (1994). Fast learning with incremental RBF networks. Neural Processing Letters, 1(1), 2-5.

Gabor, D. (1946). Theory of communication. J. IEE, 93, 429-457.

Galanopoulos, A.S., and Ahalt, S.C. (1996). Codeword distribution for frequency sensitive competitive learning with one-dimensional input data. IEEE Trans. on Neural Networks, 7, 752-756.

Gersho, A. (1979). Asymptotically optimal block quantization. IEEE Transactions on Information Processing, IT-25, 373-380.

Graepel, T., Burger, M., and Obermayer, K. (1997). Phase transitions in stochastic self-organizing maps. Physical Review E, 56(4), 3876-3890. 
Grossberg, S. (1976). Adaptive pattern classification and universal recoding: I Parallel development and coding of neural feature detectors. Biol. Cybern., 23, $121-134$.

Herbin, M., Bonnet, N., and Vautrot, P. (1996). A clustering method based on the estimation of the probability density function and on the skeleton by influence zones. Application to image processing. Pattern Recognition Letters, 17, 1141 1150.

Kim, Y.K., and Ra, J.B. (1995). Adaptive learning method in self-organizing map for edge preserving vector quantization. IEEE Trans. Neural Networks, 6, $278-280$.

Kohonen, T. (1982). Self-organized formation of topologically correct feature maps. Biol. Cybern., 43, 59-69.

Kohonen, T. (1984). Self-organization and associative memory. Heidelberg: Springer.

Kohonen, T. (1995). Self-organizing maps. Heidelberg: Springer.

Kohonen, T., Raivo, K., Simula, O., Ventä, O., and Henriksson, J. (1996). Combining linear equalization and self-organizing adaptation in dynamic discretesignal detection. Proc. IJCNN (San Diego, CA, June 17-21), pp. I223-I228.

Kohonen, T., Kaski, S., and Lappalainen, H. (1997). Self-organized formation of various invariant-feature filters in the adaptive-subspace SOM. Neural Computation 9, 1321-1344.

Lin, J.K., Grier, D.G., and Cowan, J.D. (1997). Faithful representation of separable distributions. Neural Computation, 9, 1305-1320.

Linsker, R. (1989). How to generate ordered maps by maximizing the mutual information between input and output signals. Neural Computation, 1, 402411.

Ljung, L. (1977). Analysis of recursive stochastic algorithms. IEEE Trans. Automat. Contr., AC-22, 551-575.

Moody, J., and Darken, C. (1988). Learning with localized receptive fields. Proc. 1988 Connectionist Models Summer School, D. Touretzky, G. Hinton and T. Sejnowski, eds., San Mateo: Morgan Kaufmann, pp. 133-143.

Moreau, E., and Macchi, O. (1996). High-order contrasts for self-adaptive source separation. Int'l J. of Adaptive Control and Signal Processing, 10, 19-46. 
Mulier F., and Cherkassky, V. (1995). Self-organization as an iterative kernel smoothing process. Neural Computation, 7, 1165-1177.

Nowlan, S.J. (1990). Maximum likelihood competitive learning. Advances in Neural Information Processing Systems II (Denver 1989), D.S. Touretzky (Ed.), pp. 574-582, San Mateo: Morgan Kaufmann.

Olshausen, B.A., and Field, D.J. (1996). Emergence of simple-cell receptive field properties by learning a sparse code for natural images. Nature, 381, 607-609.

Pagès, G. (1993). Voronoi tessellation, space quantization algorithms and numerical integration. Proceedings of the European Symposium on Artificial Neural Networks - ESANN'93, Brussels, Belgium, M. Verleysen (ed.), pp. 221-228.

Pajunen, P., Hyvärinen, A., and Karhunen, J. (1996). Nonlinear Blind Source Separation by Self-Organizing Maps. In S.-I. Amari, L. Xu, L.-W. Chan, I. King, and K.-S. Leung (Eds.), Progress in Neural Information Processing (Vol. 2, pp. 1207-1210). Proceedings of the International Conference on Neural Information Processing (ICONIP'96), London: Springer-Verlag.

Poggio, T., and Girosi, F. (1990). Regularization algorithms for learning that are equivalent to multilayer networks. Science, 247, 978-982.

Press, W.H., Flannery, B.P., Teukolsky, S.A., and Vetterling, W.T. (1990). Numerical recipes. The art of scientific computing. Cambridge: Cambridge University Press.

Rojas, R. (1996). Neural networks. A systematic introduction. Heidelberg: Springer.

Ritter, H., and Schulten, K. (1986). On the stationary state of Kohonen's selforganizing sensory mapping. Biol. Cybern., 54, 99-106.

Ritter, H., and Schulten, K. (1989). Combining self-organizing maps. Proc. Int. Joint Conference on Neural Networks, Vol. 2, pp. 499-502.

Ritter, H. (1991). Asymptotic level density for a class of vector quantization processes. IEEE Transactions on Neural Networks, 2(1), 173-175.

Ritter, H., Martinetz, T., and Schulten, K. (1992). Neural computation and selforganizing maps: An introduction. Reading, Mass: Addison-Wesley.

Rose, K., Gurewitz, E., and Fox, G.C. (1990). Statistical mechanics and phase transitions in clustering. Physical Review Letters, 65(8), 945-948. 
Shannon, C.E. (1960). Coding theorem for a discrete source with a fidelity criterion. In R.E. Machol (Ed.), Information and decision processes. New York, NY: McGraw-Hill.

Silverman, B.W. (1992). Density Estimation for Statistics and Data Analysis. Chapman and Hall: London.

Stone, M. (1984). Cross-validation choice and assessment of statistical predictions. J. Roy. Statist. Soc. B, 36, 111-147.

TIMIT (1990). DARPA TIMIT acoustic-phonetic speech corpus. NIST Speech Disc 1-1.1 (CD-ROM).

Ueda, N., and Nakano, R. (1993). A new learning approach based on equidistortion principle for optimal vector quantizer design. Proc. IEEE NNSP93 (Linthicum Heights, MD 1993), pp. 362-371.

Van den Bout, D. E., and Miller III, T.K. (1989). TInMANN: The integer Markovian artificial neural network. Proc. Int. Joint Conf. Neural Networks, Englewood Cliffs, N.: Erlbaum, II205-II211.

Van Hulle, M.M., and Martinez, D. (1993). On an unsupervised learning rule for scalar quantization following the maximum entropy principle. Neural Computation, 5, 939-953.

Van Hulle, M.M. (1995). Globally-ordered topology-preserving maps achieved with a learning rule performing local weight updates only. Proc. IEEE NNSP95 (Cambridge, MA 1995), pp. 95-104.

Van Hulle, M.M. (1997a). The formation of topographic maps that maximize the average mutual information of the output responses to noiseless input signals. Neural Computation, 9(3), 595-606.

Van Hulle, M.M. (1997b). Nonparametric density estimation and regression achieved with topographic maps maximizing the information-theoretic entropy of their outputs. Biol. Cybern., 77, 49-61.

Van Hulle, M.M. (1997c). Topology-preserving map formation achieved with a purely local unsupervised competitive learning rule. Neural Networks, 10(3), 431-446.

Wässle, H., Grünert, U., Röhrenbeck, J., and Boycott, B.B. (1989). Cortical magnification factor and the ganglion cell density of the primate retina. Nature, 341, 643-646. 
Xu, L., and Jordan, M.I. (1993). Unsupervised learning by EM algorithm based on finite mixture of Gaussians. World Congress on Neural Networks (Portland, OR), Vol. 2, pp. 431-434.

Zadeh, L.A. (1965). Fuzzy sets. Information and control, 8, 338-353.

Zador, P.L. (1982). Asymptotic quantization error of continuous signals and the quantization dimension. IEEE Trans. Inf. Theory, 28, 149-159. 


\section{Nomenclature}

$A$ : discrete lattice consisting of $N$ formal neurons

Act $_{i}$ : activity (activation) of neuron $i$

BDH algorithm : learning scheme introduced Bauer, Der and Herrmann (1996)

$C:$ conscience factor

$c_{i}:$ conscience of neuron $i$

$d$ : number of dimensions of $V$-space

$d_{A}:$ number of dimensions of lattice $A$

$\Delta_{i}$ : time interval between successive instants that neuron $i$ has been the winner

$E$ : energy in the original waveform

$\eta$ : learning rate

$\eta_{i}$ : learning rate of neuron $i$

FSCL : Frequency-Sensitive Competitive Learning

$f_{\text {samp }}:$ sampling frequency

$G_{c}^{i}($.$) : cosine Gabor function of neuron i$

$G_{s}^{i}($.$) : sine Gabor function of neuron i$

$i$ : neuron $i$

$i^{*}$ : winning neuron $i$

$I$ : information-theoretic entropy

kMER : kernel-based Maximum Entropy learning Rule

$K:$ (radially-symmetric) RF kernel (with center $\mathbf{w}_{i}$ and radius $\sigma_{i}$ )

$\Lambda$ : neighborhood function (in lattice space coordinates)

$M:$ number of training samples

MAE : mean absolute error

MER : (lattice-based) Maximum Entropy learning Rule (Van Hulle, 1995,1997a)

MSE : mean squared error 
$\mathcal{M}_{0}$ : score function (for smoothing purposes)

$N$ : number of formal neurons

p.d.f. : probability density function

$p(\mathbf{v})$ : input p.d.f.

$P_{i}$ : probability that neuron $i$ is active or that it is the "winner"

$P($.$) : probability$

$\tilde{p}($.$) : pilot estimate$

$\widehat{p}($.$) : Parzen window density estimate$

$\widehat{p^{*}}($.$) : fixed kernel density estimate$

$\widehat{p_{\rho_{s}}}($.$) : variable kernel density estimate with scale factor \rho_{s}$

RBF : radial basis function

RF : receptive field

RMSE : root mean squared error

$\rho$ : scale factor (for scaling kernel radii)

$\rho_{r}$ : relative scale factor $\left(\rho_{r} \doteq \frac{\rho N}{N-\rho}\right)$

$\rho_{\text {ropt }}:$ optimal $\rho_{r}$ value

$\rho_{s}$ : factor with which the radii $\sigma_{i}$ are scaled

$\rho_{\text {sopt }}:$ optimal $\rho_{s}$ value

$\sigma_{\Lambda}$ : neighborhood range (in lattice space coordinates)

$\sigma_{\Lambda 0}:$ initial neighborhood range

$\sigma_{i}$ : radius of ( $\mathrm{RF}$ region of) neuron $i$

SOM : Kohonen's Self-Organizing (feature) Map

sgn : sign function (of scalar)

Sgn : sign function of vector, acting componentwise

$S_{i}:$ RF region (hyperspherical region with radius $\sigma_{i}$ in $V$ )

$t:$ simulation time (time step) 
$t_{\max }:$ maximum number of time steps

$T$ : length of time sequence

TInMANN : The Integer Markovian Artificial Neural Network (Van den Bout and Miller, 1989)

$\tau_{i}$ : activation threshold of neuron $i$

UCL : unsupervised competitive learning rule

$V: d$-dimensional space of input signals

VK : variable kernel (method)

$\mathbf{v}=\left[v_{1}, \ldots, v_{d}\right]:$ input vector in $d$-dimensions

WTA : winner-takes-all

$\mathbf{w}_{i}=\left[w_{i 1}, \ldots, w_{i d}\right]:$ weight vector of $i$-th neuron in $d$ dimensions

$\Xi_{i}$ : fuzzy code membership function of neuron $i$

$\mathbb{1}_{i}$ : code membership function of neuron $i$ 


\section{Figure legends}

Figure 1: (A) Definition of receptive field (RF) kernel $K\left(\mathbf{v}-\mathbf{w}_{i}, \sigma_{i}\right)$ and RF region $S_{i}$. The present input $\mathbf{v} \in V$ is indicated by the black dot.

(B) The arrow indicates the update of the RF center $\mathbf{w}_{i}$, given the present input $\mathbf{v}$ (not to scale); the dashed circles indicate the updated $\mathrm{RF}$ regions $S_{i}$ and $S_{j}$. For clarity's sake, the range of the neighborhood function is assumed to have vanished so that $\mathbf{w}_{j}$ is not updated. The shaded area indicates the overlap between $S_{i}$ and $S_{j}$

Figure 2: Temporal evolution of the neuron weights (left column) and the RF regions (right column) of a $24 \times 24$ lattice with a rectangular topology using kMER. The outer squares outline the support of the uniform input distribution; the values given below the squares represent time.

Figure 3: The RF regions obtained for $\rho_{r}=0.5$ (left) and 2 (right).

Figure 4: Two-dimensional product distribution (A) and non-parametric model obtained with kMER (B), both plotted in steps of $\frac{1}{25}$. Abbreviation: $p d=$ probability density.

Figure 5: Cross-validation performance in the case of the quadrimodal product distribution example Fig. 4A (A,B), and the three equiprobable Gaussians example Fig. 6 (C,D).

(A) Three performance curves plotted as a function of $\rho_{s}$, given $\rho_{r}=1: 1$ ) the thick continuous line refers to the theoretically-expected mean squared error $M S E\left(\widehat{p_{\rho s}}, p\right)$, i.e. with reference to the analytic equation of the original distribution, 2) the thin continuous line represents our metric $M S E\left(\widehat{p_{\rho_{s}}}, \widehat{p^{*}}\right)$ eq. (25), and 3) the dashed line represents the score function $\mathcal{M}_{0}\left(\rho_{s}\right)$ eq. (21). For the sake of representation only, the score function $\mathcal{M}_{0}$ is shifted upwards by 0.8 .

(B) The corresponding curves when $\rho_{r}$ is also minimized, in addition to $\rho_{s}$, plotted as a function of $\rho_{r}$.

(C) The performance curves plotted as a function of $\rho_{s}$, given $\rho_{r}=17.5$. Again for representational purposes, the score function is shifted upwards by 2.0 and the result divided by 10 .

(D) The performance curves plotted as a function of $\rho_{r}$, except for the dashed 
curve which shows the score function $\mathcal{M}_{0}$ for $\rho_{s}=1$ (see text). The score function is shifted upwards by 1.0 and divided by 25 .

Figure 6: Scatter plot of three times 300 samples drawn from three equallyprobable Gaussians (pluses, crosses, and stars).

Figure 7: Topographic feature map formation applied to speech signals.

(A) Example of a speech signal taken from the TIMIT database.

(B) Energy spectrum of the speech signal, plotted in terms of radian frequencies.

(C) Temporal evolution of the kernel weights when trained with kMER for $\rho=2$.

(D) The kernels obtained at convergence, plotted in the radian frequency domain.

(E) The energy spectrum estimate that corresponds to (D) (thick line) vs. the original spectrum (thin line).

(F) The energy spectrum estimate for $\rho=8$ (thick line) vs. the original spectrum (thin line).

Figure 8: The wavelet filters (cosine Gabors functions) obtained with kMER. The abscissa spans $30 \mathrm{~ms}$. 


\section{Tables}

Table 1: Equiprobabilistic map performance obtained for the quadrimodal product distribution example, using different learning rules in incremental learning mode.

\begin{tabular}{l|c|c}
\hline rule & RMSE & $I$ \\
\hline SOM & $9.49 \times 10^{-4}$ & 8.95 \\
MER & $8.06 \times 10^{-4}$ & 9.04 \\
BDH & $2.34 \times 10^{-3}$ & 8.36 \\
FSCL & $5.20 \times 10^{-4}$ & 9.10 \\
Conscience 1 & $5.91 \times 10^{-4}$ & 9.08 \\
Conscience 2 & $3.58 \times 10^{-4}$ & 9.14 \\
kMER & $2.75 \times 10^{-4}$ & 9.15 \\
\hline
\end{tabular}

Table 2: Equiprobabilistic map performance for the three equally-probable Gaussians example and batch learning.

\begin{tabular}{l|c|c}
\hline rule & RMSE & $I$ \\
\hline SOM & $8.11 \times 10^{-4}$ & 9.01 \\
MER & $8.05 \times 10^{-4}$ & 9.02 \\
BDH & $1.51 \times 10^{-3}$ & 8.77 \\
FSCL & $5.60 \times 10^{-4}$ & 9.10 \\
Conscience 1 & $5.39 \times 10^{-5}$ & 9.17 \\
Conscience 2 & $5.38 \times 10^{-5}$ & 9.17 \\
kMER & $4.81 \times 10^{-5}$ & 9.17 \\
\hline
\end{tabular}


Table 3: Density estimation performance for the product distribution example, expressed in MSE and Kullback-Leibler (KL) distance, for different learning rules and the VK method.

\begin{tabular}{l|c|l}
\hline rule/method & MSE & KL distance \\
\hline SOM & $1.21 \times 10^{-1}$ & 226.8 \\
MER & $6.63 \times 10^{-2}$ & 55.0 \\
BDH & $8.98 \times 10^{-2}$ & 290.6 \\
FSCL & $8.42 \times 10^{-2}$ & 98.0 \\
Conscience 1 & $7.25 \times 10^{-2}$ & 66.1 \\
Conscience 2 & $6.93 \times 10^{-2}$ & 51.5 \\
kMER & $5.76 \times 10^{-2}$ & 30.5 \\
VK & $5.35 \times 10^{-2}$ & 21.4 \\
\hline
\end{tabular}

Table 4: Density estimation performance for the three equally-probable Gaussians example, for different learning rules and the VK and Parzen/histogram methods.

\begin{tabular}{l|c|l}
\hline rule/method & MSE & KL distance \\
\hline SOM & $1.75 \times 10^{-2}$ & 48.2 \\
MER & $1.34 \times 10^{-2}$ & 35.1 \\
BDH & $2.58 \times 10^{-2}$ & 273.8 \\
FSCL & $1.29 \times 10^{-2}$ & 37.6 \\
Conscience 1 & $9.63 \times 10^{-3}$ & 27.6 \\
Conscience 2 & $9.37 \times 10^{-3}$ & 27.3 \\
kMER & $7.72 \times 10^{-3}$ & 25.9 \\
VK & $7.66 \times 10^{-3}$ & 22.9 \\
Parzen/histogram & $2.55 \times 10^{-2}$ & 82.3 \\
\hline
\end{tabular}


Table 5: Time-complexity of the learning algorithms used in the simulations, expressed in $N M d$ units and listed as a function of the number of assignments $(=)$, additions/subtractions $(+/-)$ and multiplications/divisions (*/:). For MER's time-complexity, see text.

\begin{tabular}{l|ccc}
\hline \multirow{2}{*}{ rule } & \multicolumn{2}{|c}{ time-complexity } \\
\cline { 2 - 4 } & $=$ & $+/-$ & $* /:$ \\
\hline SOM & 2 & 4 & 2 \\
MER & - & - & - \\
BDH & 2 & 4 & 3 \\
FSCL & 2 & 4 & 2 \\
Conscience 1 & 2 & 4 & 2 \\
Conscience 2 & 2 & 5 & 3 \\
kMER & 3 & 3 & 2 \\
\hline
\end{tabular}



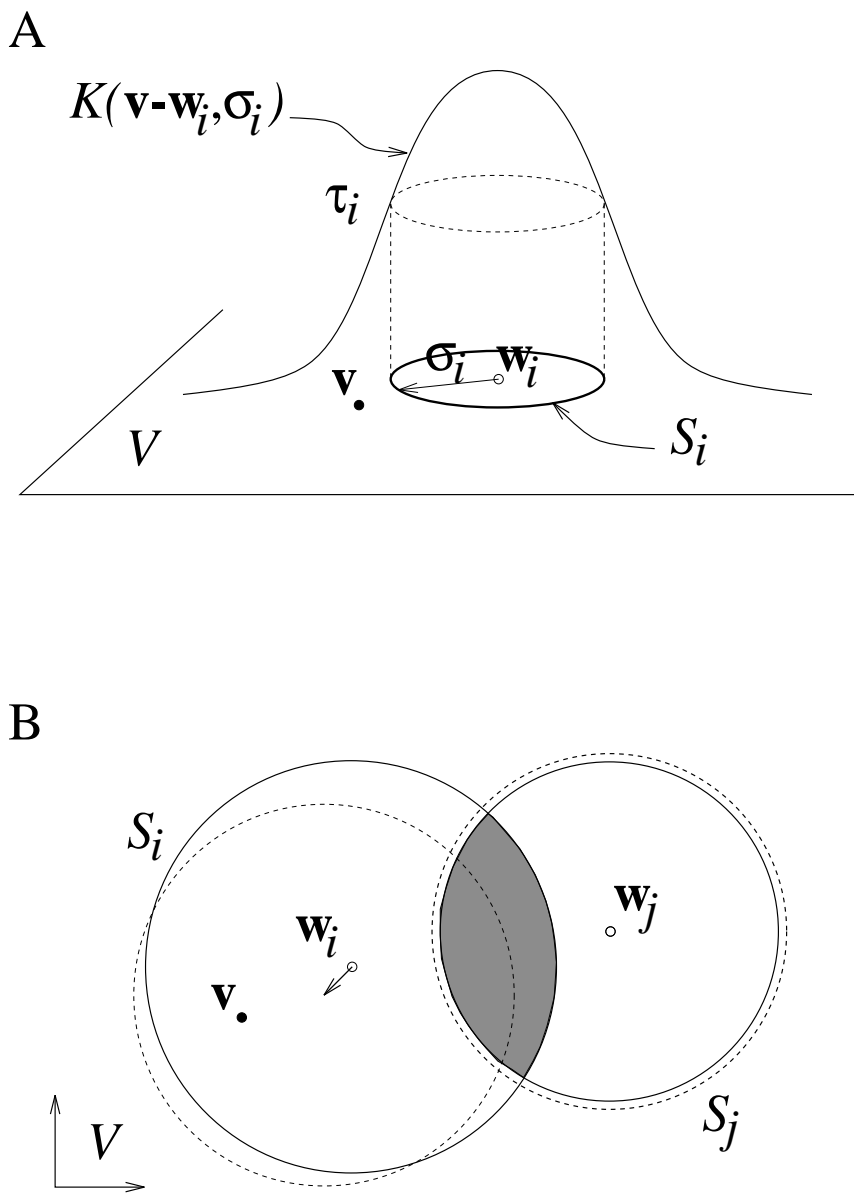

Figure 1: 

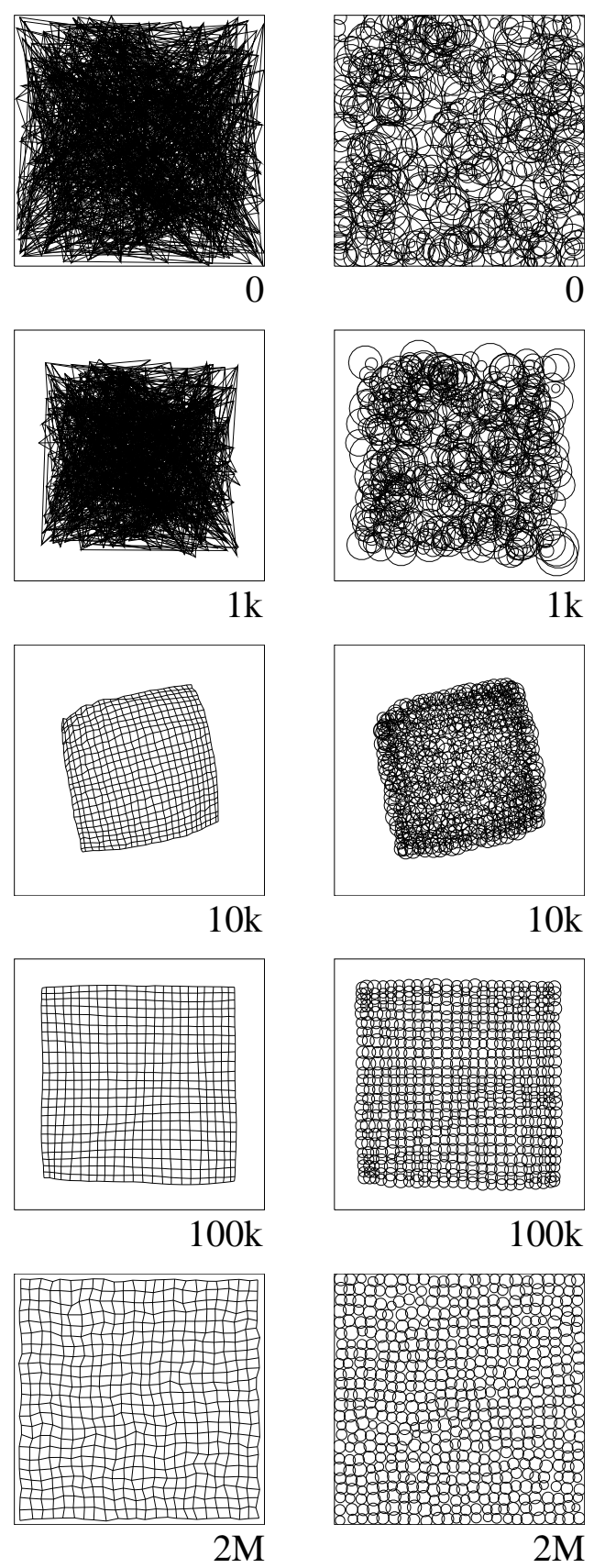

Figure 2: 

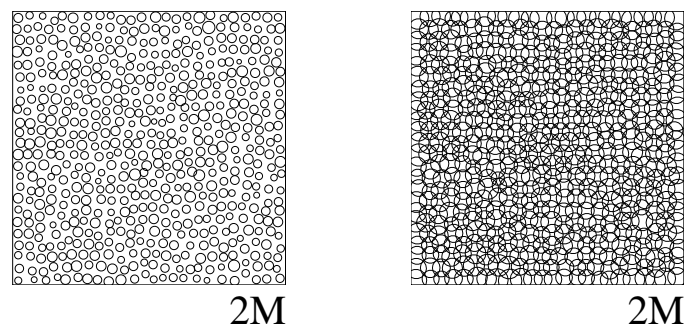

Figure 3: 
A

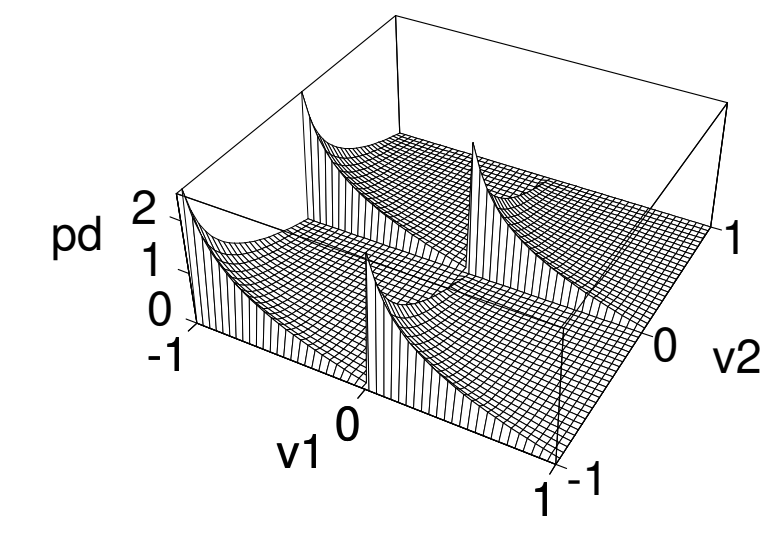

B

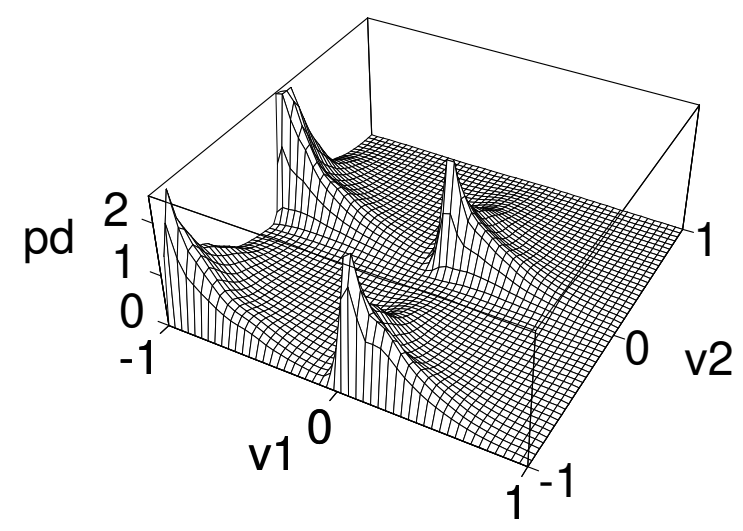

Figure 4: 

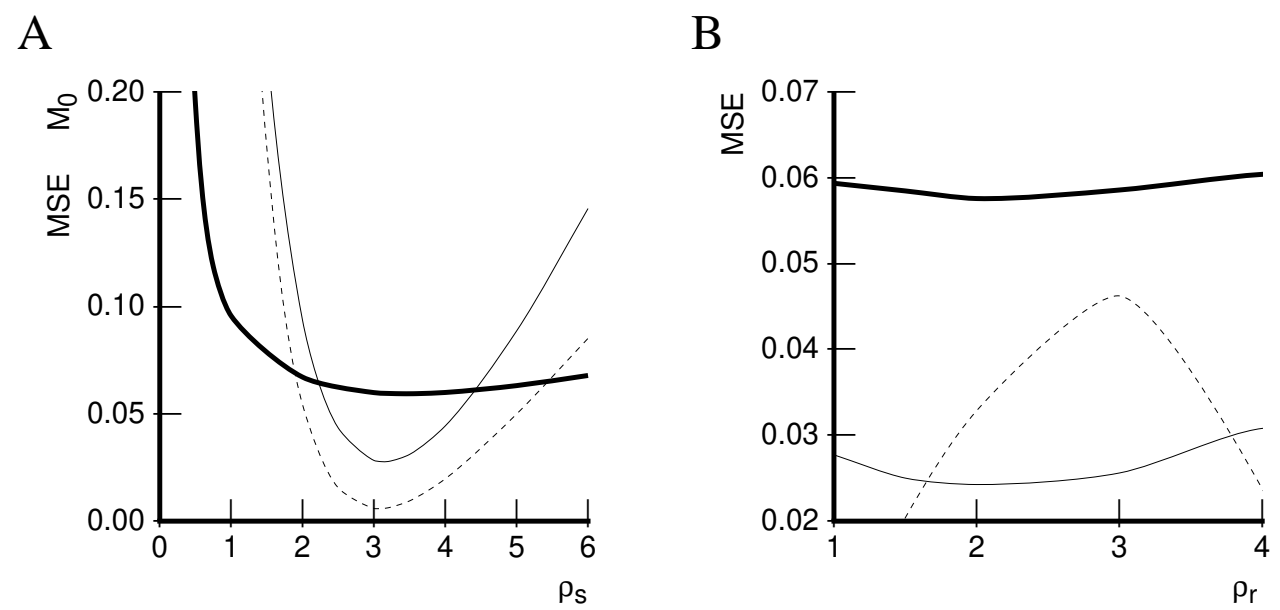

C

D
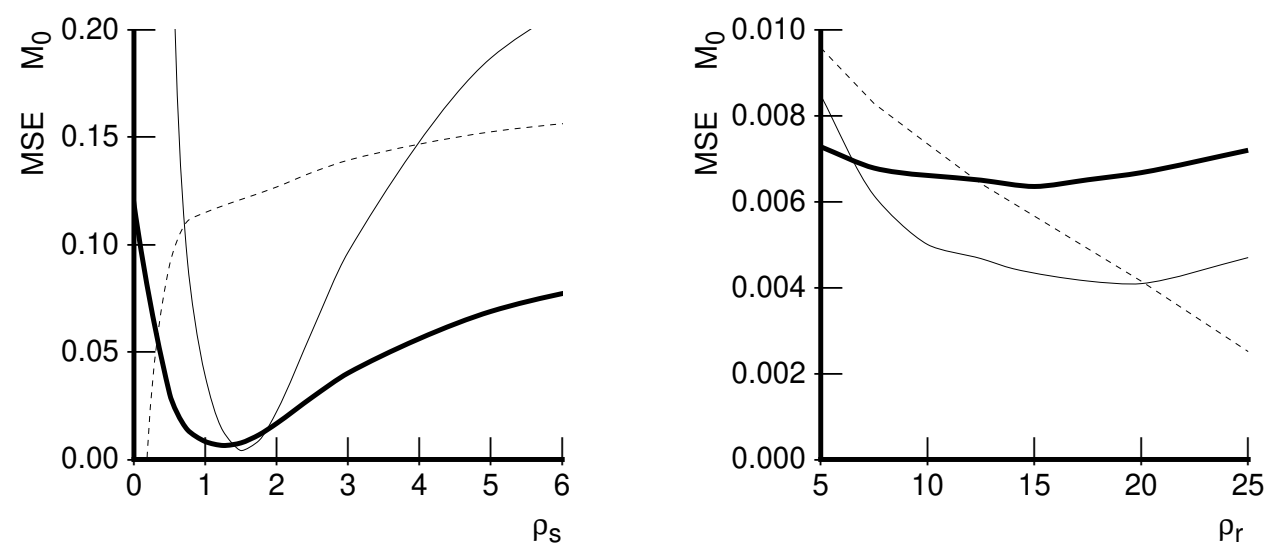

Figure 5: 


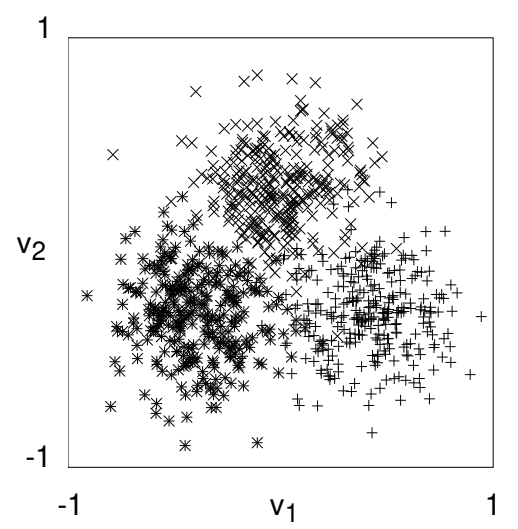

Figure 6: 
A

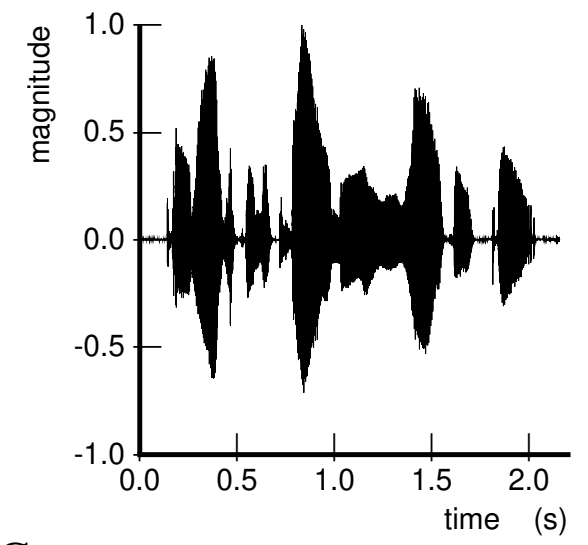

C

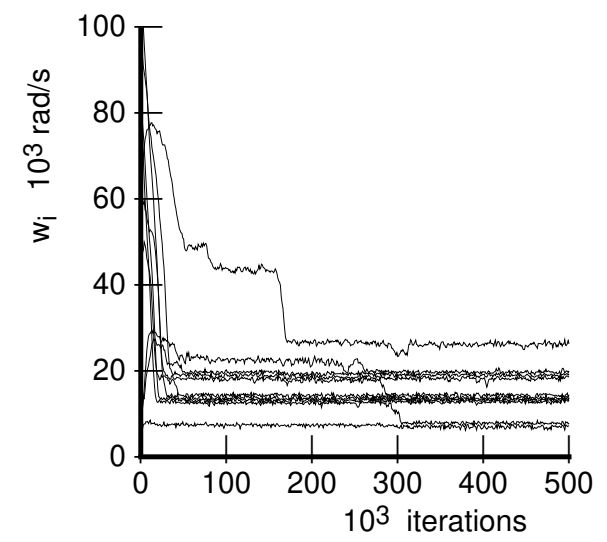

E

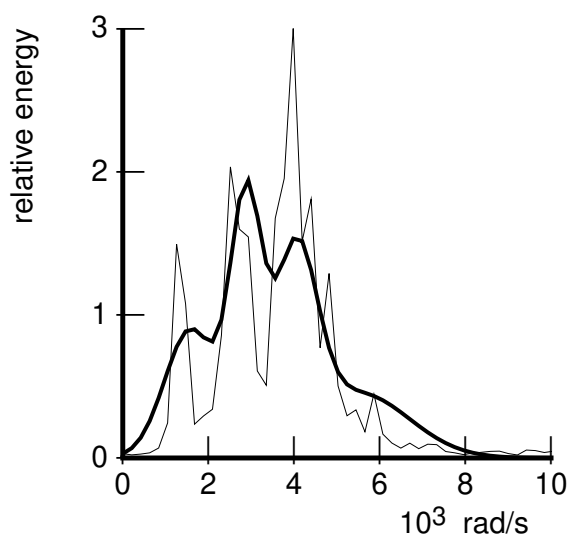

B

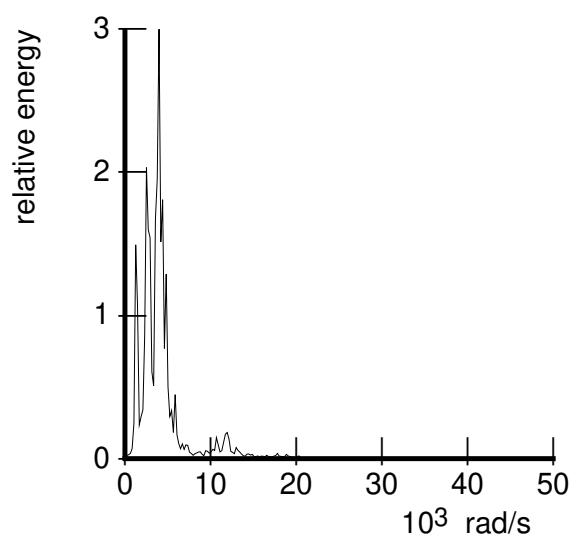

D

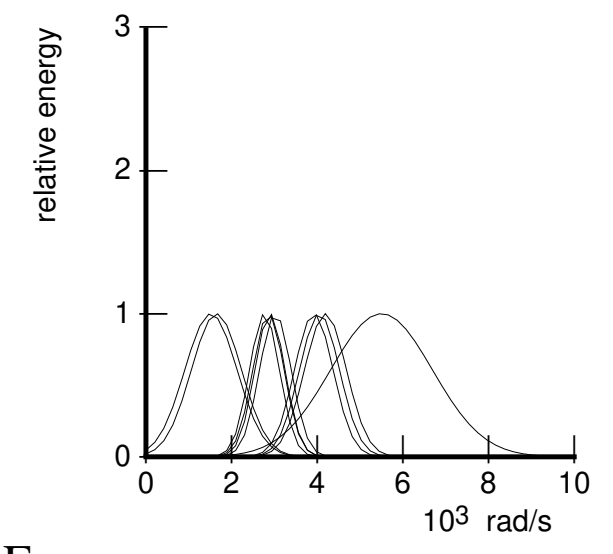

F

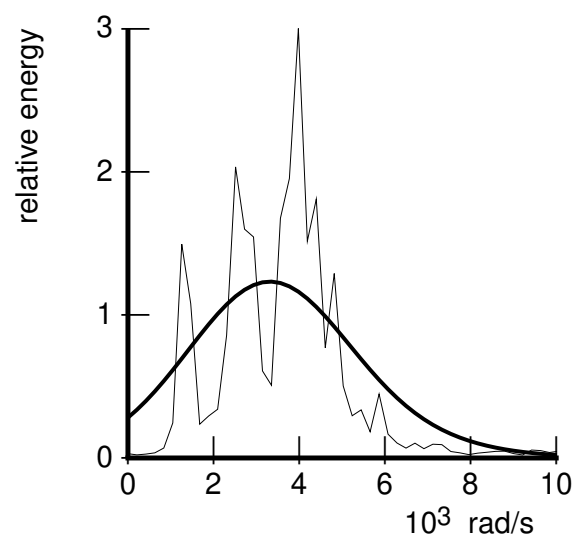

Figure 7: 


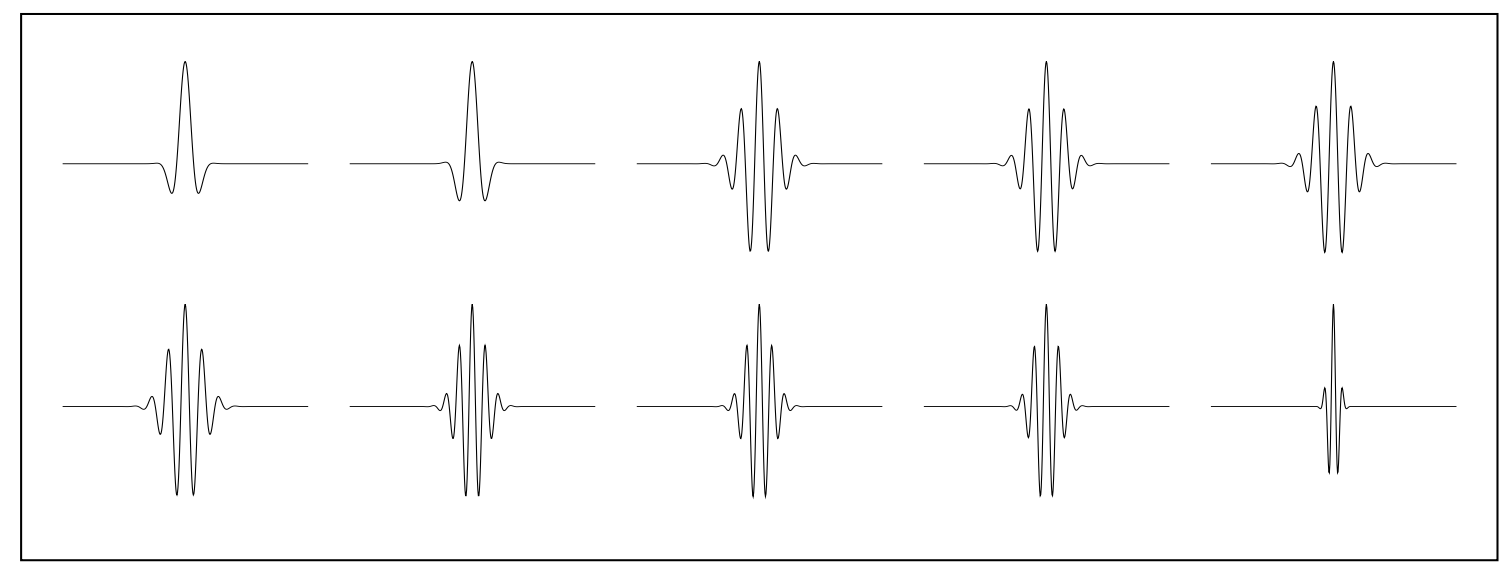

Figure 8: 
$\begin{array}{ll}1 & 1\end{array}$

$\begin{array}{ll}2 & 2\end{array}$

$\begin{array}{ll}3 & 3\end{array}$

$\begin{array}{ll}4 & 4\end{array}$

55

$\begin{array}{ll}6 & 6\end{array}$

$\begin{array}{ll}7 & 7\end{array}$ 